\title{
METALLOGENIC MAP OF ZINC, LEAD, AND BARIUM DEPOSITS AND OCCURRENCES IN PALEOZOIC SEDIMENTARY ROCKS, EAST-CENTRAL UNITED STATES
}

\author{
By Sandra H. B. Clark
}

\section{INTRODUCTION}

This pamphlet, which accompanies map I-1773, is a descriptive compilation of 545 zinc, lead, and barium districts, deposits, and occurrences in Paleozoic sedimentary rocks in the east-central United States. Numbers generally are assigned to the deposits and occurrences in a left to right and top to bottom pattern within each state (listed alphabetically). The numbers correspond to the numbers within each state on the accompanying map. More than one name is given for localities that have been called by different names at different times. The name listed first is that most recently or commonly used in the literature, or is from a nearby geographical locality. Other names for the occurrences are listed in parenthesis. Names of subdistricts and (or) some of the larger mines are listed in parentheses after district names. These names are included to aid the reader in identifying localities as listed in other reports and are not necessarily current. This is especially true for names of quarries, which may or may not be currently operating or being operated by the same company.

Commodities are listed in order of abundance, if known, in the order listed in previous reports, or, if there is no other information, $\mathrm{Pb}$ and $\mathrm{Zn}$ before $\mathrm{Ba}$. Minor or byproduct commodities are in parentheses. Commodities are shown by chemical symbols: $\mathrm{Pb}$, lead; $\mathrm{Zn}$, zinc; $\mathrm{Ba}$, barium; $\mathrm{Cu}$, copper; $\mathrm{F}$, fluorine; $\mathrm{As}$, arsenic; $\mathrm{U}$, uranium; $\mathrm{Ag}$, silver; $\mathrm{Cd}$, cadmium. Size is given as occurrence or prospect or as small, medium or large for deposits which have been mined. Definitions of small, medium and large are those used by Guild (1981, p. A4). Stratigraphic names and geologic ages are taken from the references cited and do not necessarily conform to the usage of the U.S. Geological Survey. References listed are selected to provide information on the specific deposit or occurrence shown, but are not comprehensive listings of references to each deposit or occurrence. The compilation is generally restricted to deposits and mineral occurrences that have been described in the literature, although data for a few occurrences are from unpublished sources. Dashes indicate unknown or no information available.

\section{CONTENTS}

State

Number of Locations

Page

2
Alabama

9

Georgia

9

Illinois

33

Indiana

148

Kentucky

Maryland

Michigan

New York

Ohio

Pennsylvania

Tennessee

3

1

8

34

71

77

56

Vermont

Virginia

West Virginia

4

6

References cited 


\begin{tabular}{|c|c|c|c|c|c|c|}
\hline $\begin{array}{c}\text { Map } \\
\text { number }\end{array}$ & $\underline{\text { Name }}$ & Commodities & $\underline{\text { Size }}$ & Host rock & Comments & $\begin{array}{c}\text { Selected } \\
\text { references } \\
\end{array}$ \\
\hline 4 & $\begin{array}{l}\text { Angel District } \\
\text { (Evans mine, } \\
\text { Angel Station) }\end{array}$ & $\mathrm{Ba}$ & Sma 11 & $\begin{array}{l}\text { In residual soil over- } \\
\text { lying Cambrian and Lower } \\
\text { Ordovician dolomite and } \\
\text { limestone (Copper Ridge, } \\
\text { Chepultepec, Newala, and } \\
\text { Longview Formations) }\end{array}$ & $\begin{array}{l}\text { Fracture filling and } \\
\text { replacement; boxwork } \\
\text { patterns. }\end{array}$ & $\begin{array}{l}\text { Adams and Jones, } \\
1940, \text { p. 34-37; } \\
\text { Hughes and Lynch, } \\
1973 \text {, p. 34-41. }\end{array}$ \\
\hline 6 & Leeds area & $\mathrm{Ba}$ & do. & $\begin{array}{l}\text { In residual clay } \\
\text { overlying Lower } \\
\text { Ordovician Newala } \\
\text { Limestone }\end{array}$ & $\begin{array}{l}\text { Calcite veins and } \\
\text { irregular vein-like } \\
\text { replacement of barite } \\
\text { in limestone. }\end{array}$ & $\begin{array}{l}\text { Adams and Jones, } \\
1940, \text { p. 17-18, 32; } \\
\text { Hughes and Lynch, } \\
1973 \text {, p. } 31-32 \text {. }\end{array}$ \\
\hline 7 & $\begin{array}{l}\text { Vincent, } \\
\text { Harpersville, } \\
\text { Wilsonville } \\
\text { district }\end{array}$ & $\mathrm{Ba}$ & do. & $\begin{array}{l}\text { In residuum of Cambrian } \\
\text { and Lower Ordovician } \\
\text { dolomite and limestone }\end{array}$ & $\begin{array}{l}\text { Barite occurs in } \\
\text { residuum of the } \\
\text { Copper Ridge and } \\
\text { Chepultepec } \\
\text { Formations, but } \\
\text { may have been } \\
\text { derived from } \\
\text { weathering of the } \\
\text { overlying limestone. }\end{array}$ & $\begin{array}{l}\text { Adams and Jones, } \\
1940 \text {, p. 17-18, } \\
30-31 \text {; Hughes and } \\
\text { Lynch, 1973, } \\
\text { p. } 30-31 \text {. }\end{array}$ \\
\hline
\end{tabular}


Map

number

Name

Commodities

$\mathrm{Ba}$

8

Longview Saginaw district (Longview

Lime works)

9 Sinks District

$\mathrm{Ba}(\mathrm{F})$

(Julian Fancher,

D.C. Weaver,

Herron, Hardy

Pratt)

\section{Host rock}

In residual clay overlying Lower Ordovician Newala Limestone

In residual clay overlying Ordovician dolomite or limestone (Newala Limestone and Mosheim Limestone)

\section{Comments}

In one quarry

brecciated Newala

Limestone is cemented

and partly replaced

by calcite. Barite

occurred immediately

above this horizon.

As veins in limestone. Fluid inclusions have a temperature of $70^{\circ} \mathrm{C}$ $92^{\circ} \mathrm{C}$ (uncorrected for pressure). Veins contain calcite,

marcasite, sphalerite, goethite, and massive sulfur.
Adams and Jones, 1940 , p. 31;

Hughes and Lynch, 1973, p. 29-30.

Adams and Jones, 1940, p. 21-29; Hughes and Lynch, 1973, p. 24-29; Jones and McVay, 1934. 


\begin{tabular}{|c|c|c|c|c|c|c|}
\hline $\begin{array}{c}\text { Map } \\
\text { number }\end{array}$ & $\underline{\text { Name }}$ & Commodities & $\underline{\text { Size }}$ & Host rock & Comments & $\begin{array}{l}\text { Selected } \\
\text { references }\end{array}$ \\
\hline 1 & $\begin{array}{l}\text { Hale dolomite } \\
\text { quarry }\end{array}$ & $\mathrm{Ba}, \mathrm{F}, \mathrm{Pb}$ & Occurrence & $\begin{array}{l}\text { Ordovician dolomite } \\
\text { (Knox Group) }\end{array}$ & $\begin{array}{l}\text { In a five-foot thick } \\
\text { brecciated zone. }\end{array}$ & $\begin{array}{l}\text { Butts and } \\
\text { Gildersleeve, } \\
1948, \text { p. } 111\end{array}$ \\
\hline 2 & $\begin{array}{l}\text { Ruralville } \\
\text { area }\end{array}$ & $\mathrm{Ba}$ & Sma11 & $\begin{array}{l}\text { In soil which contains } \\
\text { fragments from } \\
\text { Ordovician Knox Group }\end{array}$ & $\begin{array}{l}\text { Residual deposit of } \\
\text { white crystalline } \\
\text { barite in yellow to } \\
\text { reddish-yellow clay } \\
\text { loam. }\end{array}$ & $\begin{array}{l}\text { Hul1, } 1920, \text { p. 134- } \\
136 .\end{array}$ \\
\hline 3 & Eton district & $\mathrm{Ba}$ & do. & $\begin{array}{l}\text { In red soil which } \\
\text { overlies Ordovician } \\
\text { Knox Group }\end{array}$ & $\begin{array}{l}\text { Residual deposit with } \\
\text { white crystalline barite } \\
\text { and some dark fragments } \\
\text { with radiating structure. }\end{array}$ & $\begin{array}{l}\text { Hul1, } 1920, \text { p. 129- } \\
\text { 133. }\end{array}$ \\
\hline 4 & Plainville area & $\mathrm{Ba}$ & do. & $\begin{array}{l}\text { In brown soil and } \\
\text { in Cambrian limestone } \\
\text { (Conasauga Formation) }\end{array}$ & $\begin{array}{l}\text { Residual deposits and } \\
\text { veins in underlying } \\
\text { limestone. }\end{array}$ & $\begin{array}{l}\text { Hu 11, 1920, p. 136- } \\
139 .\end{array}$ \\
\hline 5 & Big Dry Creek & $\mathrm{Pb}$ & Occurrence & Limestone & $\begin{array}{l}\text { Small galena cubes } \\
\text { have been found in a } \\
\text { limestone quarry. }\end{array}$ & $\begin{array}{l}\text { Cook, 1978, p. 24; } \\
\text { Hurst and Crawford, } \\
1970, \text { p. } 147 .\end{array}$ \\
\hline 6 & Gibson property & $\mathrm{Ba}$ & Prospect & $\begin{array}{l}\text { Residual in soil } \\
\text { which contains } \\
\text { weathered seams of } \\
\text { Ordovician Knox } \\
\text { dolomite }\end{array}$ & $\begin{array}{l}\text { Fragments in dark red } \\
\text { soil, thought to be } \\
\text { derived from veins or } \\
\text { fracture fillings. }\end{array}$ & $\begin{array}{l}\text { Hul1, 1920, p. 139- } \\
140 .\end{array}$ \\
\hline 7 & $\begin{array}{l}\text { Holcombe } \\
\text { property }\end{array}$ & $\mathrm{Ba}$ & Sma 11 & $\begin{array}{l}\text { In soil overlying } \\
\text { Ordovician dolomite } \\
\text { (Knox Group) }\end{array}$ & Fragments in red clay. & $\begin{array}{l}\text { Hu11, } 1920, \text { p. } 127- \\
129 .\end{array}$ \\
\hline 8 & $\begin{array}{l}\text { Cartersville } \\
\text { district }\end{array}$ & $\mathrm{Ba}(\mathrm{Mn}, \mathrm{Fe})$ & Large & $\begin{array}{l}\text { In soils; primary } \\
\text { ore-bearing strata } \\
\text { are Lower Cambrian } \\
\text { dolomite (Rome and } \\
\text { Shady Formations) }\end{array}$ & $\begin{array}{l}\text { As white to very light- } \\
\text { blue, fine-grained, } \\
\text { granular masses in } \\
\text { residuum and in veins, } \\
\text { pods and fracture } \\
\text { fillings. Residual } \\
\text { clays up to } 200 \text { feet } \\
\text { thick. }\end{array}$ & $\begin{array}{l}\text { Kesler, 1950; } \\
\text { Reade and others, } \\
1980 .\end{array}$ \\
\hline
\end{tabular}




\section{Map}

number

Name

Stilesboro

area

\section{Commodities}

$\mathrm{Ba}$
Host rock

In soil overlying

Ordovician 1 imestone

(Chickamauga Formation)
Selected

references

Fragments in reddishyellow soil.

Hu 11, 1920, p. 126 127. 
Map

number

Name

Commodities

$\mathrm{Zn}, \mathrm{Pb}$

Upper Mississipp

Valley lead-zin

district (The

district includes

about 35 lead and

zinc mines in

Illinois. Sub-

districts are

Elizabeth, Apple

River-Warren,

Stockton, Simmons

Mound, Morseville,

Scales Mound, and

Galena).

This locality is

the same as site 1

of Wisconsin.

area

Stadermann gold mine (Eleroy area)

$\mathrm{Au}, \mathrm{Cu}$,

$\mathrm{Pb}, \mathrm{Zn}, \mathrm{Ba}$
Freeport area
$(\mathrm{Cu}, \mathrm{Ba})$

$\underline{\text { Size }}$

Host rock

Large

Middle Ordovician

limestone and dolomite

(most deposits in

Galena Dolomite,

Decorah Formation, and

Platteville Formation); deposits of sulfides

found in all formations

exposed within the

mineralized part of

the district.

\section{Middle Ordovician}

Galena Dolomite

do.

Upper Ordovician

Maquoketa Shale

$\mathrm{Pb}$

do. Middle Ordovician

\section{Comments}

Ore bodies can be

classified as (1) reverse-

fault and fold controlled

ore bodies, (2) joint-

controlled ore bodies,

and (3) placer and residual deposits. The ores in the

fault and fold deposits

occur as (1) vein fillings

along fractures and bedding

planes; (2) cavity fillings

in solution breccias; and

(3) disseminations by

replacement and impregna-

tion in favorable beds,

particularly in shaly

strata. Joint controlled

deposits are veins in

vertical joints and pod-

like deposit in beds

crossed by joints (Hey 1

and others, 1959).

Gash veins.

Small veins and lenses contain galena, sphalerite and barite; gold-bearing quartz veins in dolomite.
Se lected

references

Bradbury, 1959;

Hey1 and others, 1959; Hey 1 , 1968a; Heyi and West, 1982:

McLimans, 1977.

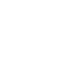

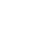

Heyl and others, 1959, p. 293-294; Hey 1 and West, 1982 , p. 1804 .

Hey 1 and others, 1959, p. 293-294; Hey 1 and West, 1982 , p. 1804.

Heyl and others, 1959, p. 293; Heyl and West, 1982 , p. 1804; Willman and others, 1946 , p. 9. 


\begin{tabular}{|c|c|c|c|c|c|c|}
\hline $\begin{array}{l}\text { Map } \\
\text { number }\end{array}$ & Name & Commodities & $\underline{\text { Size }}$ & Host rock & Comments & $\begin{array}{l}\text { Selected } \\
\text { references }\end{array}$ \\
\hline 5 & $\begin{array}{l}\text { Yellow Creek } \\
\text { or Stebbins lead } \\
\text { mine area }\end{array}$ & $\mathrm{Pb}$ & do. & $\begin{array}{l}\text { Middle Ordovician } \\
\text { Galena Dolomite and } \\
\text { Platteville Formation }\end{array}$ & Mined before 1920 . & $\begin{array}{l}\text { Hey } 1 \text { and West, } 1982 \text {, } \\
\text { p. 1804, 1807. }\end{array}$ \\
\hline 6 & -- & $\mathrm{Pb}$ & Occurrence & -- & -- & $\begin{array}{l}\text { Heyl and others, } \\
1959 \text {, p. } 293 \text {. }\end{array}$ \\
\hline 7 & -- & $\mathrm{Pb}$ & do. & -- & -- & Do. \\
\hline 8 & $\begin{array}{l}\text { Mt. Carrol1 } \\
\text { diggings }\end{array}$ & $\mathrm{Pb}$ & Small & $\begin{array}{l}\text { Middle Ordovician } \\
\text { Galena Dolomite }\end{array}$ & $\begin{array}{l}\text { In gash veins and } \\
\text { openings; thousands } \\
\text { of shailow workings. }\end{array}$ & $\begin{array}{l}\text { Heyl and others, } \\
1959 \text {, p. 293; Hey } 1 \\
\text { and West, 1982, } \\
\text { p. } 1804,1807 \text {; } \\
\text { Wil lman and others, } \\
\text { 1946. }\end{array}$ \\
\hline 9 & -- & $\mathrm{Pb}$ & do. & -- & -- & Heyl and West, 1982. \\
\hline 10 & -- & $\mathrm{Pb}$ & do. & -- & -- & Do. \\
\hline 11 & -- & $\mathrm{Pb}$ & Occurrence & -- & -- & Do. \\
\hline 12 & -- & $\mathrm{Pb}$ & do. & -- & -- & Do. \\
\hline 13 & -- & $\mathrm{Zn}$ & do. & -- & - & Do. \\
\hline 14 & $\begin{array}{l}\text { Quarry of Midway } \\
\text { Stone Company }\end{array}$ & $\mathrm{Zn}$ & Occurrence & $\begin{array}{l}\text { Silurian dolomite } \\
\text { (Racine Formation) }\end{array}$ & $\begin{array}{l}\text { Sphalerite with pyrite } \\
\text { and marcasite on joint } \\
\text { faces in fracture zone. }\end{array}$ & $\begin{array}{l}\text { J. C. Bradbury, } \\
\text { written Commun. 1983; } \\
\text { Hey } 1 \text {, 1968b; Hey } 1 \\
\text { and West, 1982, } \\
\text { p. } 1804 .\end{array}$ \\
\hline 15 & $\begin{array}{l}\text { Mecca Mine No. } 1 \text {, } \\
\text { Midland Electric } \\
\text { Coal Corporation }\end{array}$ & $\mathrm{Zn}$ & Occurrence & $\begin{array}{l}\text { Pennsylvanian coal } \\
\text { (Carbondale Group, } \\
\text { No. } 6 \text { coal) }\end{array}$ & $\begin{array}{l}597 \mathrm{ppm} \mathrm{Zn} \text { in ash; } \\
\text { ident ified as sphalerite. }\end{array}$ & Zubovic, 1960. \\
\hline
\end{tabular}


Map

number

16

Name

Commodities

Millbrook, near

$\mathrm{Zn}(\mathrm{Pb})$

Barron Mill farm

Size

do.

\section{Host rock}

Middle Ordovician

Galena-Platteville

Dolomite

LaSalle Stone

Company quarry

(abandoned)

Essex Mine Northern Illinois

Coal Corporation

19

Allentown Mine, Midland Coa

Company

Mecco Mine

(eastern and

western pits)

Midland Coal

Company

21

Midland Coal

Company

(western pit)

Edwards Mine,

Midland Coa 1

Company

Norris Mine,

Consolidated

Coal Company

Sun Spot Mine, AMAX Coal

Company
$\mathrm{Ba}$

\section{Pennsylvanian LaSalle} Limestone

$\mathrm{Zn}$ do.

Pennsylvanian coal

(Carbondale Group,

No. 5 coal)

do. Coal

do.

do.

do.

do.

do.

do.

do.

do.

do.

do.

\section{Comments}

Sphalerite at a depth of 60-62 feet in a water well; interpreted as

residue in a solution

cavity along a fault or

joint within the Sandwich

fault zone.

As cavity fillings.

$415 \mathrm{ppm} \mathrm{Zn}$ in ash.

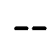

$-$
Bradbury, 1957, p. 4; Payne, 1938.
Schrode, 1952.

Zubovic, 1960.

Cobb, 1981;

Cobb and others, 1980 .

Do.

Do.

Do.

Do.

Do. 
Buckheart Mine

(southern pit),

Freeman United

Coal Mining Company

Big Eagle No. 1

Mine, Great

American Energy

Corporation

Crown No. 2 Mine,

Freeman United Coal

Company

Peabody Coa 1

Company No. 17

Mine

do.

do.

$--$

Do.

do.

do.

do.

do.

do. Pennsylvanian Coal

(Carbondale Group No. 6 coal)

Grand Tower

$\mathrm{Pb}$

31

Hutchins Creek

$\mathrm{Pb}, \mathrm{Zn}(\mathrm{Cu})$

(near Alto Pass)

do.

Devonian limestone Limestone)

\section{Devonian 1 imestone}

(Backbone Limestone and Clear Creek Chert)

Along a fault; found during railroad

construction.

Sma 11 crystals

disseminated in

1 imestone; along

faults.

Occurrence Coal
$\mathrm{Zn}$

Eagle No. 2

Surface Mine,

Peabody Coa 1

Company 
Vein deposits, which are along normal faults

related to horsts and grabens; bedded deposits; solution-slump breccias, which extend downward

below some bedded deposits; and diatremes, which are related to

the Hicks dome structure (Pinckney, 1976).
Grogan and Bradbury 1968; Hey 1 and others, 1965 .

Pinckney, 1976; Trace, 1976; Wor 1 and others, 1974 , p. 4-5 and map. 


\section{INDIANA}

\begin{tabular}{|c|c|c|c|c|c|c|}
\hline $\begin{array}{l}\text { Map } \\
\text { number }\end{array}$ & $\underline{\text { Name }}$ & Commodities & $\underline{\text { Size }}$ & Host rock & Comments & $\begin{array}{l}\text { Selected } \\
\text { references }\end{array}$ \\
\hline 1 & -- & $\mathrm{Zn}$ & 0ccurrence & $\begin{array}{l}\text { Middle Ordovician } \\
\text { dolomite (Trenton } \\
\text { Limestone) }\end{array}$ & In we 11 cuttings. & $\begin{array}{l}\text { Shaffer, 1981, } \\
\text { p. } 30 .\end{array}$ \\
\hline 2 & $\mathrm{Z22} 1 /$ & Zn & do. & do. & $\begin{array}{l}\text { In well cuttings; } \\
\text { sphalerite shows for } \\
70 \text { feet. }\end{array}$ & Do. \\
\hline 3 & $\mathrm{Z23}$ & $\mathrm{Zn}$ & do. & do. & In we1l cuttings. & Do. \\
\hline 4 & $Z 20 A$ and $20 B$ & $\mathrm{Zn}, \mathrm{Pb}$ & do. & do. & do. & Do. \\
\hline 5 & $\mathrm{Z25}$ & $\mathrm{Zn}$ & do. & do. & do. & Do. \\
\hline 6 & $\mathrm{Z21}$ & $\mathrm{Zn}$ & do. & $\begin{array}{l}\text { Devonian dolomite } \\
\text { (Muscatatuck Group) }\end{array}$ & do. & Do. \\
\hline 7 & Z38 & $\mathrm{Zn}$ & do. & $\begin{array}{l}\text { Middle Ordovician silty } \\
\text { dolomite (Trenton } \\
\text { Limestone) }\end{array}$ & do. & $\begin{array}{l}\text { Shaffer, 1981, } \\
\text { p. } 31 \text {. }\end{array}$ \\
\hline 8 & Z34A and $34 B$ & $\mathrm{Zn}$ & do. & $\begin{array}{l}\text { Middle Ordovician dolomite } \\
\text { (Trenton Limestone) }\end{array}$ & do. & Do. \\
\hline 9 & -- & $\mathrm{Zn}, \mathrm{Pb}$ & do. & do. & do. & $\begin{array}{l}\text { Shaffer, 1981, } \\
\text { p. } 30 .\end{array}$ \\
\hline 10 & 217 and 218 & $\mathrm{Zn}$ & do. & $\begin{array}{l}\text { Silurian dolomite } \\
\text { (Salamonie Dolomite) }\end{array}$ & do. & Do. \\
\hline 11 & Z16 & $\mathrm{Zn}$ & do. & $\begin{array}{l}\text { Devonian limestone } \\
\text { (Muscatatuck Group) }\end{array}$ & do. & Do. \\
\hline 12 & Z84 & $\mathrm{Zn}, \mathrm{Pb}$ & do. & $\begin{array}{l}\text { Middle Ordovician } \\
\text { dolomite (Trenton } \\
\text { Limestone?) }\end{array}$ & $\begin{array}{l}\text { Quarry exposures at the } \\
\text { Kentland cryptoexplosion } \\
\text { structure. }\end{array}$ & $\begin{array}{l}\text { Shaffer, 1981, } \\
\text { p. } 32,35 .\end{array}$ \\
\hline
\end{tabular}

1/ Sample Numbers, Shaffer, 1981, p. 30-32. 


\begin{tabular}{|c|c|c|c|c|c|c|}
\hline $\begin{array}{l}\text { Map } \\
\text { number }\end{array}$ & Name & Commodities & $\underline{\text { Size }}$ & Host rock & Comments & $\begin{array}{l}\text { Selected } \\
\text { references }\end{array}$ \\
\hline 13 & $\begin{array}{l}\text { Babcock } \\
\text { Construction } \\
\text { Company quarry }\end{array}$ & $\mathrm{Zn}$ & do. & -- & In quarry. & $\begin{array}{l}\text { Erd and Greenberg, } \\
1960, p .50,51,55 ; \\
\text { Shaffer, } 1981, \\
\text { p. } 32 .\end{array}$ \\
\hline 14 & -- & $\mathrm{Zn}, \mathrm{Pb}$ & do. & $\begin{array}{l}\text { Devonian limestone } \\
\text { (Muscatatuck Group) }\end{array}$ & Surface exposure. & $\begin{array}{l}\text { Shaffer, 1981, } \\
\text { p. } 32 \text {. }\end{array}$ \\
\hline 15 & $\mathrm{Z15}$ & $\mathrm{Zn}$ & do. & do. & In we 11 cuttings. & $\begin{array}{l}\text { Shaffer, 1981, } \\
\text { p. } 30 .\end{array}$ \\
\hline 16 & Z35 & $\mathrm{Zn}$ & do. & $\begin{array}{l}\text { Silurian dolomite } \\
\text { (Wabash Formation) }\end{array}$ & do. & $\begin{array}{l}\text { Shaffer, 1981, } \\
\text { p. } 31 \text {. }\end{array}$ \\
\hline 17 & -- & $\mathrm{Zn}$ & do. & $\begin{array}{l}\text { Silurian limestone } \\
\text { (Salamonie Dolomite) }\end{array}$ & do. & $\begin{array}{l}\text { Shaffer, 1981, } \\
\text { p. } 30 .\end{array}$ \\
\hline 18 & $\mathrm{Z7}$ and $\mathrm{Z53}$ & $\mathrm{Zn}$ & do. & $\begin{array}{l}\text { Middle Ordovician } \\
\text { dolomite (Trenton } \\
\text { Limestone) }\end{array}$ & do. & Do. \\
\hline 19 & 219 & $\mathrm{Zn}$ & do. & $\begin{array}{l}\text { Silurian dolomite } \\
\text { (Salamonie Dolomite) }\end{array}$ & do. & Bo. \\
\hline 20 & -- & $\mathrm{Zn}$ & do. & $\begin{array}{l}\text { Devonian limestone } \\
\text { (Muscatatuck Formation) }\end{array}$ & do. & $\begin{array}{l}\text { Shaffer, } 1981 \text {, } \\
\text { p. } 31 \text {. }\end{array}$ \\
\hline 21 & $\mathbf{Z 5 0}$ & $\mathrm{Zn}$ & do. & $\begin{array}{l}\text { Silurian dolomite } \\
\text { (Wabash Formation) }\end{array}$ & do. & Do. \\
\hline 22 & 251 & $\mathrm{Zn}$ & do. & do. & do. & Do. \\
\hline 23 & -- & $\mathrm{Zn}$ & do. & $\begin{array}{l}\text { Devonian limestone } \\
\text { (Muscatatuck Formation) }\end{array}$ & do. & Do. \\
\hline 24 & $248 A$ and $48 B$ & $\mathrm{Zn}$ & do. & do. & do. & Do. \\
\hline 25 & $\begin{aligned} Z 55, & 56,57 \\
68,69 & \end{aligned}$ & $\mathrm{Zn}$ & do. & $\begin{array}{l}\text { Silurian dolomite } \\
\text { (Wabash Formation) }\end{array}$ & do. & Do. \\
\hline 26 & $\mathrm{Z76}$ & $\mathrm{Zn}$ & do. & do. & do. & Do. \\
\hline
\end{tabular}


\begin{tabular}{c} 
Map \\
number \\
\hline
\end{tabular}

27

28

29

Eric Stone Company quarry

Z1

Heller Stone Company quarry

\section{$\mathrm{Zn}$}

$\mathrm{Zn}$

$$
\mathrm{Zn}, \mathrm{Pb}
$$

Zn

$\mathrm{Zn}$ $\mathrm{Zn}$ $\mathrm{Zn}$

Zn

Zn

$\mathrm{Zn}$

\section{Size}

do.

Upper Ordovician shaly limestone (Maquoketa Group)

do.

do.

Middle Ordovician dolomite (Trenton Limestone)

do.

do. Devonian 1 imestone (Muscatatuck Group)

do.

Silurian dolomite (Wabash Formation) do.

Middle Ordovician dolomite (Trenton Limestone)

do. Middle Ordovician dolomite (Trenton Formation)

do.

do. Silurian dolomite (Wabash Formation)

do. do. do. do.

Outcrop along Big Pipe Creek 1 mile north of Bunker Hill.

In we11 cuttings.

Surface exposure.

do.

do.

do.

do.

Do.

Do.

Erd and Greenberg, p. 50, 51, 63; Shaffer, 1981, p. 25.

Shaffer, 1981, p. 32 .

Shaffer, 1981, p. 31 . 


\begin{tabular}{|c|c|c|c|c|c|c|}
\hline $\begin{array}{c}\text { Map } \\
\text { number }\end{array}$ & Name & Commodities & $\underline{\text { Size }}$ & Host rock & Comments & $\begin{array}{c}\text { Selected } \\
\text { references }\end{array}$ \\
\hline 39 & -- & $\mathrm{Ba}$ & do. & -- & Surface exposure. & $\begin{array}{l}\text { Shaffer, 1981, } \\
\text { p. } 25 .\end{array}$ \\
\hline 40 & -- & $\mathrm{Ba}$ & do. & -- & do. & $\begin{array}{l}\text { Shaffer, } 1981 \text {, } \\
\text { p. } 25 .\end{array}$ \\
\hline 41 & $\begin{array}{l}\text { Bluff a long } \\
\text { Mud Pine Creek }\end{array}$ & $\mathrm{Zn}, \mathrm{Ba}$ & do. & -- & do. & $\begin{array}{l}\text { Erd and Greenberg, } \\
1960 \text {, p. } 18,19,50 \text {, } \\
51,62 \text {; Shaffer, } \\
1981, \text { p. } 25 .\end{array}$ \\
\hline 42 & -- & $\mathrm{Zn}$ & do. & -- & $\begin{array}{l}\text { From a small coal } \\
\text { mine. }\end{array}$ & $\begin{array}{l}\text { Erd and Greenberg, } \\
1960, \text { p. } 50,51,62 \text {; } \\
\text { Shaffer, 1981, } \\
\text { p. } 25 \text {. }\end{array}$ \\
\hline 43 & Z41 & $\mathrm{Zn}$ & do. & $\begin{array}{l}\text { Middle Ordovician } \\
\text { dolomite (Black } \\
\text { River Formation) }\end{array}$ & In we11 cuttings. & $\begin{array}{l}\text { Shaffer, 1981, } \\
\text { p. } 31 .\end{array}$ \\
\hline 44 & $\mathrm{Z77}$ & $\mathrm{Zn}$ & do. & $\begin{array}{l}\text { Silurian dolomite } \\
\text { (Wabash Formation) }\end{array}$ & Surface exposure. & $\begin{array}{l}\text { Shaffer, 1981, } \\
\text { p. } 32 \text {. }\end{array}$ \\
\hline 45 & -- & $\mathrm{Zn}$ & do. & do. & In well cuttings. & $\begin{array}{l}\text { Shaffer, 1981, } \\
\text { p. } 30 .\end{array}$ \\
\hline 46 & Z42 & $\mathrm{Zn}$ & do. & $\begin{array}{l}\text { Middle Ordovician } \\
\text { dolomite (Trenton } \\
\text { Limestone) }\end{array}$ & do. & $\begin{array}{l}\text { Shaffer, 1981, } \\
\text { p. 31. }\end{array}$ \\
\hline 47 & -- & $\mathrm{Ba}$ & do. & -- & Surface exposure. & $\begin{array}{l}\text { Shaffer, } 1981 \text {, } \\
\text { p. } 38 .\end{array}$ \\
\hline 48 & Z80 & $\mathrm{Zn}$ & do. & $\begin{array}{l}\text { Devonian dolomite } \\
\text { (Muscatatuck Group) }\end{array}$ & Surface exposure. & $\begin{array}{l}\text { Shaffer, } 1981 \text {, } \\
\text { p. } 32 \text {. }\end{array}$ \\
\hline 49 & $\mathbf{Z 7 3}$ & $\mathrm{Zn}$ & do. & do. & do. & Do. \\
\hline 50 & $\mathrm{Z12}$ & $\mathrm{Zn}$ & do. & $\begin{array}{l}\text { Middle Ordovician } \\
\text { dolomite (Trenton } \\
\text { Limestone) }\end{array}$ & In we 11 cuttings. & $\begin{array}{l}\text { Shaffer, 1981, } \\
\text { p. } 30 .\end{array}$ \\
\hline 51 & $\mathrm{Z37}$ & $\mathrm{Zn}$ & do. & $\begin{array}{l}\text { Lower Ordovician } \\
\text { dolomite (Knox Group) }\end{array}$ & do. & $\begin{array}{l}\text { Shaffer, 1981, } \\
\text { p. 31. }\end{array}$ \\
\hline
\end{tabular}




\begin{tabular}{|c|c|c|c|c|c|}
\hline $\begin{array}{c}\text { Map } \\
\text { number }\end{array}$ & Name & Commodities & $\underline{\text { Size }}$ & Host rock & Comments \\
\hline 52 & $\mathrm{Z36}$ & $\mathrm{Zn}$ & do. & do. & do. \\
\hline 53 & DeBolt quarry & $\mathrm{Zn}, \mathrm{Ba}$ & do. & -- & -- \\
\hline 54 & $\begin{array}{l}\text { Abandoned } \\
\text { quarry }\end{array}$ & $\mathrm{Ba}$ & do. & -- & -- \\
\hline 55 & -- & $\mathrm{Zn}$ & do. & -- & Surface exposure. \\
\hline 56 & $\mathrm{Z31}$ & $\mathrm{Zn}$ & do. & $\begin{array}{l}\text { Mississippian lime- } \\
\text { stone (Sanders } \\
\text { Formation) }\end{array}$ & In well cuttings. \\
\hline 57 & $\begin{array}{l}\text { Wallace quarry } \\
\text { (abandoned) }\end{array}$ & $\mathrm{Zn}, \mathrm{Ba}$ & do. & -- & Surface exposure. \\
\hline 58 & $\begin{array}{l}\text { Waveland Stone } \\
\text { Company quarry }\end{array}$ & $\mathrm{Zn}, \mathrm{Ba}$ & do. & -- & -- \\
\hline 59 & $\begin{array}{l}\text { Russellville } \\
\text { Stone Company } \\
\text { quarry }\end{array}$ & $\mathrm{Zn}, \mathrm{Ba}$ & do. & -- & -- \\
\hline 60 & $\begin{array}{l}\text { Abandoned } \\
\text { quarry }\end{array}$ & $\mathrm{Zn}$ & do. & -- & -- \\
\hline 61 & $\begin{array}{l}\text { Parkersburg } \\
\text { quarry } \\
\text { (abandoned) }\end{array}$ & $\mathrm{Zn}$ & do. & -- & -- \\
\hline
\end{tabular}

Do.

Erd and Greenberg, 1960 , p. 18-19, 50 , 51, 63; Shaffer, 1981 , p. 25, 26.

Erd and Greenberg, 1960, p. 18-19, 63; Shaffer, 1981, p. 26 .

Shaffer, 1981, p. 25 .

Shaffer, 1981, p. 31 .

Erd and Greenberg, 1960 , p. 18, 19, $50,51,60$; Shaffer 1981 , p. 25.

Erd and Greenberg 1960 , p. 18, 19, 50 , 51, 59; Shaffer, 1981 , p. 25.

Erd and Greenberg, 1960 , p. 18, 19, 50 , 51, 60; Shaffer, 1981, p. 25.

Erd and Greenberg, 1960 , p. 50, 51, 61; Shaffer, 1981, p. 25.

Erd and Greenberg, 1960 , p. 50, 51, 59 Shaffer, 1981, p. 25 . 


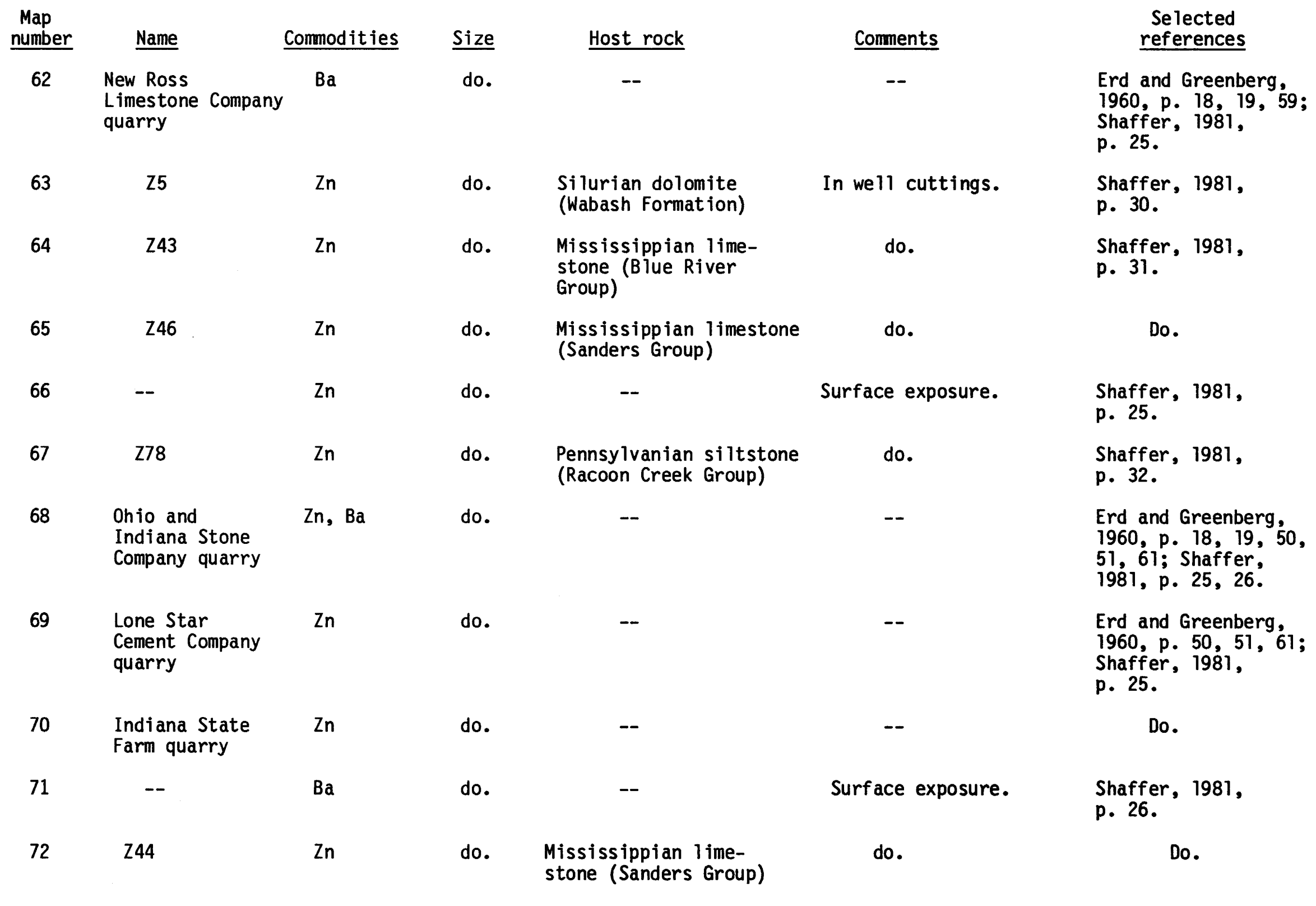


do.

Pennsylvanian coa 1 (Seelyville Coal III)

74

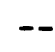

$\mathrm{Zn}$

Zn

Zn

$76 \quad 245$

77

78

Bicknel 1 Coal

Company

79

80

28

81

82

29

83

Z10

$\mathbf{Z 5 2}$

85

\section{Zn}

Zn

Zn

Zn

$\mathrm{Zn}$

Zn

$\mathrm{Zn}$

$\mathrm{Zn}$ do. Pennsylvanian shale

do.

do.

do. Mississippian 1imestone (Blue River Group)

do. Devonian dolomite (Muscatatuck Group)

do. Sandstone

do. Mississippian limeGroup)

do. Silurian? limestone

(Wabash Formation?)

do. Mississippian limestone (Sanders Group)

do. Mississippian limestone (BTue River Group)

do. Devonian dolomite (Muscatatuck Group)

do.

do.

do.
(Racoon Creek Group) stone (Blue River

\section{Comments}

Pyrite and marcasite are the main sulfides: sphalerite is rarely present, occurring in fusinite associated with cleat pyrite.

do.

do.

do.

do.

In siderite

concretions.

In we 11 cuttings.

do.

do.

do.

do.

do.

Outcrop along McCormicks Creek.
Boctor and others, 1976.

Shaffer, 1981, p. 31 .

Do.

Do.

Do.

Erd and Greenberg 1960 , p. $50,51,56$; Shaffer, 1981, p. 25 .

Shaffer, 1981, p. 30 .

Do.

Do.

Do.

Do.

Do.

Erd and Greenberg 1960 , p. 50, 51, 60; Shaffer, 1981, p. 25. 

Company Pit

$\mathrm{Zn}, \mathrm{Ba}$

$\mathrm{Ba}$

$\mathrm{Zn}$

$-\quad \mathrm{Zn}, \mathrm{Ba}$

do.

do.

do.

do.

Devonian dolomite (Muscatatuck Group)

do. do. do.

Mississippian limestone (Sanders Group)

do.

do.

do.

\section{Devonian dolomite} (Muscatatuck Group)
Comments

In cut near railroad station.

In cut on secondary road.

In abandoned quarry.

In we 11 cuttings.

In well cuttings.

do.

In road cut on Indiana 37 .

In well cuttings. Near (not shown separately) of sphalerite in Mississippian limestone (Sanders Group).

In abandoned quarry. a surface exposure 271

Erd and Greenberg,

1960 , p. 50, 51, 59;

Shaffer, 1981, p. 25.

Erd and Greenberg,

1960 , p. 50, 51, 59;

Shaffer, 1981, p. 25.

Erd and Greenberg

1960 , p. $18,19,50$,

51, 60; Shaffer,

1981 , p. 25, 26.

Shaffer, 1981, p. 31 .

Erd and Greenberg,

1960 , p. 18, 19, 59;

Shaffer, 1981, p. 25

Shaffer, 1981, p. 31 .

Erd and Greenberg, 1960 , p. 18, 19, 50, 51, 57; Shaffer, 1981 , p. 25.

Erd and Greenberg, $1960,18,19,57$; Shaffer, 1981, p. 25

Shaffer, 1981, p. 31,32 .

Erd and Greenberg, $1960,18,19,50$. 51, 57; Shaffer, 1981 , p. 25 . 


\section{do.}

Devonian dolomite (Mascatatuck Group)

97

Z6

98

Bloomington

Crushed Stone

Company quarry

100

Griffys Creek

$\mathrm{Zn}$

$\vec{\omega}$

101

102

$--$

$\mathrm{Zn}, \mathrm{Ba}$

$\mathrm{Zn}, \mathrm{Ba}$
Zn

$\mathrm{Zn}$

\section{Silurian dolomite} (Salamonie Dolomite)

do.

do.

do.

do.

do.

do.

do.

do.

$\mathrm{Ba}$

$--$

(Muscatatuck Group)

do.
Outcrop on sma 11

tributary to

Griffys Creek.

Outcrop and wash

in stream.

do.

In abandoned quarry.

Exposure in temporary excavation.

do.

In road cut on

Shaffer, 1981, p. 31.

Shaffer, 1981, p. 30.

Erd and Greenberg,

1960 , p. 18, 19,

$50,51,57$; Shaffer, 1981 , p. 25.

Erd and Greenberg, 1960 , p. 18, 19, 50, 51, 58; Shaffer, 1981 , p. 25.

Erd and Greenberg, 1960, p. 50, 51, 57; Shaffer, 1981, p. 25.

Erd and Greenberg, 1960 , p. 50, 51, 58; Shaffer, 1981, p. 25.

Do.

Erd and Greenberg, 1960 , p. 18, 19, 50, 51, 57; Shaffer, 1981 , p. 25.

Erd and Greenberg, 1960 , p. 50, 51, 58; Shaffer, 1981, p. 25 .

Do. Indiana 46.

Erd and Greenberg, 1960 , p. 18, 19, 58; Shaffer, 1981, p. 25. 


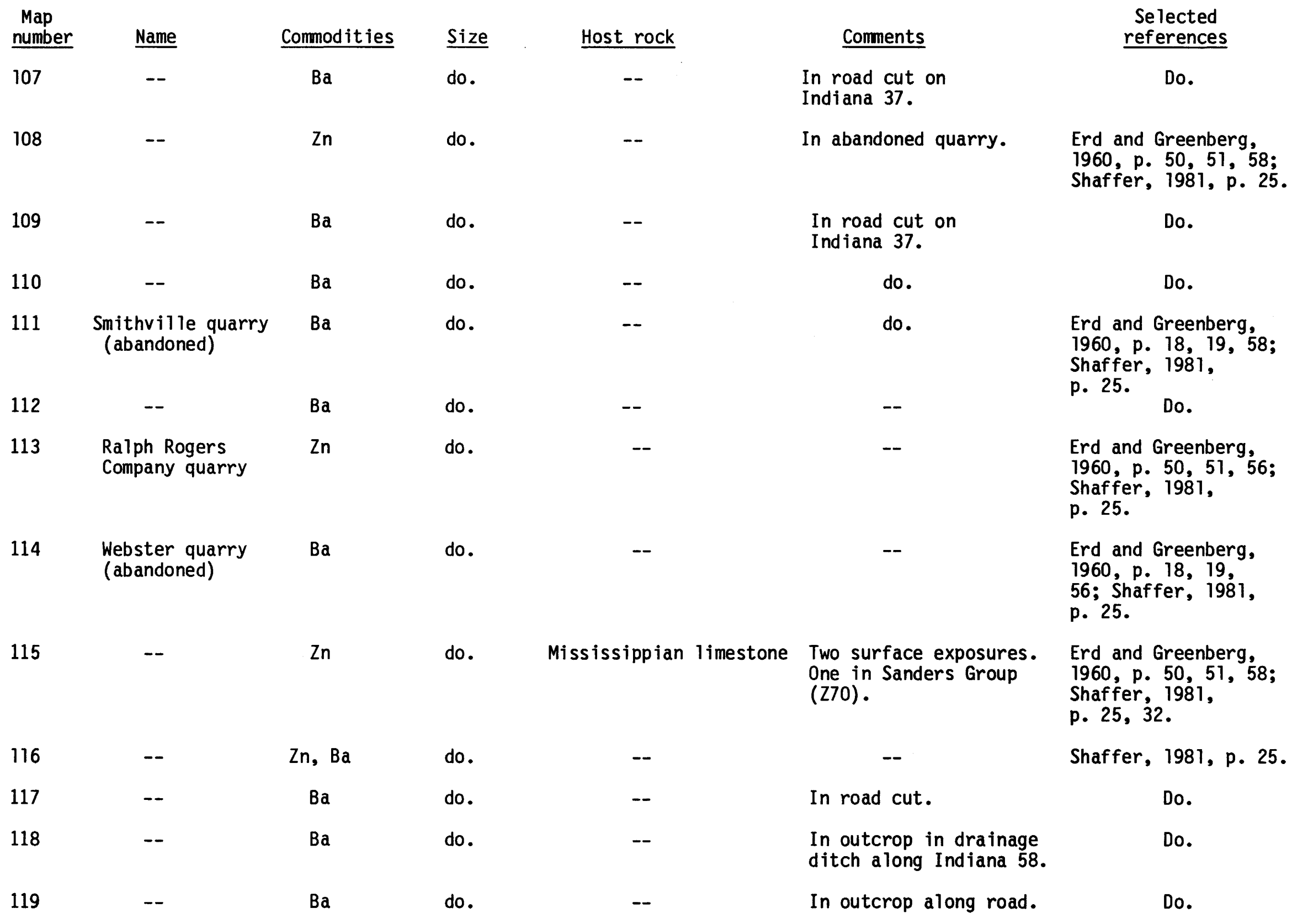




\section{Map}

number

Name

Commodities

$\mathrm{Zn}$

$\mathrm{Zn}$

121

281

122

Nally, Ballard, and Cuto quarry

123

$$
--\quad \mathrm{Zn}, \mathrm{Ba}
$$

$\mathrm{Ba}$

124

Radcliff and

Berry, Inc.

quarry

125

126

$--\quad \mathrm{Zn}, \mathrm{Ba}$

127

Seymour Grave 1
Company quarry

$\mathrm{Zn}, \mathrm{Ba}$

128

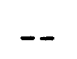

$\mathrm{Zn}$

$\mathrm{Zn}$

$--\quad$ Zn

Host rock

Mississippian limestone (Sanders Group)

do.

Mississippian limestone

(Blue River Group)

do.

do.

$--$

do.

do.

$-$

do.

Mississippian

Harrodsburg

Limestone (?)

do.

\section{Mississippi}

Harrodsburg Limestone

do.

Mississippian limestone (Salamonie ? Dolomite)

Comments

In well cuttings.
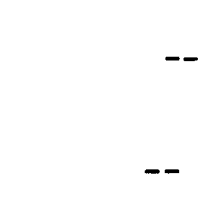

In abandoned quarry and railroad cut.

\section{$-$}

In abandoned quarry.

From an abandoned quarry immediately west of Seymour Gravel Company quarry (Indiana 127).

In geodes.

In we11 cuttings.

do. Devonian limestone

(Muscatatuck Group)
Se lected

references

Shaffer, 1981, p. 30.

Shaffer, 1981, p. 32.

Erd and Greenberg,

1960 , p. 18, 19, 57;

Shaffer, 1981,

p. 25 .

Erd and Greenberg, 1960 , p. 18, 19, 50, 51,56 ; Shaffer, 1981 , p. 25.

Erd and Greenberg, 1960 , p. 18, 19, 59; Shaffer, 1981, p. 25.

Erd and Greenberg, 1960 , p. 18, 19, 62; Shaffer, 1981, p. 26.

Erd and Greenberg, 1960 , p. 18, 19, 50, 51, 55; Shaffer, 1981 , p. 25.

Do.

Shaffer, 1981, p. 30 .

Shaffer, 1981, p. 32 . 
Stone Company quarry

$$
\mathrm{Z74}
$$

$\mathrm{Zn}$

do.

do.

132

Paul Frank

$\mathrm{Zn}, \mathrm{Ba}$

do.

$$
-
$$

Scott County Stone Company quarry quarry

137 Salem Lime and Stone Company quarry (abandoned)

138 Hoosier Lime and quarry Stone Company

$$
\mathrm{Zn}, \mathrm{Ba}
$$

\section{Zn}

Zn -- do.

do.

$B a$

Zn

$\mathrm{Ba}$

do.

do.

do.

do.

do.
In road cut on Indiana 129.

In railroad cut.

In cut for dam spillage.
Erd and Greenberg, 1960 , p. 50, 51, 53; Shaffer, 1981.

Do.

Erd and Greenberg, 1960 , p. 18, 19, 50, 51, 55; Shaffer, 1981 , p. 25.

Erd and Greenberg, 1960 , p. 18, 19, 61; Shaffer, 1981, p. 26.

Erd and Greenberg, 1960 , p. 50, 51, 61; Shaffer, 1981, p. 25.

Erd and Greenberg, 1960 , p. 18, 19, 55; Shaffer, 1981, p. 25.

Erd and Greenberg, 1960 , p. 18, 19, 62; Shaffer, 1981, p. 26.

Erd and Greenberg, 1960 , p. 50, 51, 62; Shaffer, 1981, p. 25.

Do.

Erd and Greenberg, 1960 , p. 18, 19, 50, 51, 62; Shaffer, 1981 , p. 25,26 . 


\section{Map \\ number}

140

141

142 do.

do.

do.

do.

do.

do.

do.

do.

do.

Host rock

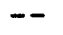

$--$

$-$

$-$

$--$

Mississippian limestone (Sanders Group)

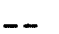

Comments

In railroad cut.

In road cut on Indiana Route 60.

In abandoned quarry.

Surface exposure.

In well cuttings.

In road cut on Indiana 70 .

In well cuttings.
Selected

references

Erd and Greenberg 1960 , p. 50, 51, 62; Shaffer, 1981, p. 25.

Erd and Greenberg, 1960 , p. 50, 51, 63; Shaffer, 1981, p. 25 .

Erd and Greenberg, 1960 , p. 50, 51, 63; Shaffer, 1981, p. 25 .

Erd and Greenberg, 1960, p. $18,19,50$, 51, 63; Shaffer, 1981 , p. 25,26 .

Shaffer, 1981, p. 25.

Shaffer, 1981, p. 30 .

Erd and Greenberg, 1960 , p. $50,51,61$; Shaffer, 1981, p. 25 .

Erd and Greenberg, 1960 , p. 18, 19, 60; Shaffer, 1981, p. 26 .

Shaffer, 1981 , p. 31 . 


\section{Map}

number

1

Central Kentucky

district (More

than 200 vein

deposits; 177

mines and

prospects

described by

Robinson, 1931)

2 Cumber land River area (Central

Tennessee

district)

3

(I11-

Illinois-

Kentucky

nois fluorspar

33) district (In

Kentucky more

than 120

mines, mine

groups, pros-

pects, and

occurrences

in the

Western

Kentucky

district)

\section{Commodities Size \\ Host rock \\ Comments}

$\mathrm{Ba}(\mathrm{Zn}, \mathrm{Pb}, \mathrm{F})$ Medium

Mostly in Middle Ordovician limestone;

$96 \%$ of the veins are

in Lexington Limestone

Formation, Tyrone

Limestone, and lower

Clay's Ferry Formation.

Some veins extend into

Upper Ordovician

Richmond Group.

$\mathrm{Ba}, \mathrm{F}, \mathrm{Zn}, \mathrm{Pb}$ Prospects

Upper Ordovician

limestone (Leipers

Limestone) and

Mississippian shale

and limestone (Fort

Payne Formation)

$\mathrm{F}(\mathrm{Zn}, \mathrm{Pb}, \mathrm{Ba})$ Medium Mississippian limezinc-lead stone, shale, and production. sandstone

Large

fluorspar

production.
In steeply dipping veins and fault breccia fillings on the Jessamine dome. Most veins are in nearly vertical strikeslip faults.

Steeply dipping veins and fault breccia fillings; veinlets of barite in shale; barite in geodes and as irregular nodular segregations in 1 imestone.

Vein deposits, bedding replacement deposits, and residual deposits; most large vein deposits are in the principal

NE- or E-trending

faults; some are in cross or oblique faults

or other fractures. Some veins are fissure

fillings; others have

resulted from replacement of wall rock. In some deposits

fluorspar cements

breccia fragments.
Se lected

references

Jolly and Heyl, 1964: Plummer, 1971; Fohs, 1913; Robinson, 1931.

Jolly and Heyl, 1964; Plummer, 1971; Taylor,

1962 .

Currier, 1923, 1937; Grogan and Bradbury, 1968: Pinckney,

1976; Williams and others, 1954; Wor 1 and others, 1974, p. 5 and map. 
MARYLAND

Map

number

1 Sauble quarry, Johnsville

\section{$\underline{\text { Size }}$ \\ Occurrence}

Host rock

Cambrian(?) Wakef ield Marble
Comments

Lumps, small bands and stringers in

brecciated and crushed marble.
Se lected references

Brobst, 1965, p. 5; Hey 1 and Pearre, 1965, p. 40,

fig. 2; 0strander, 1942, Watson and Grasty, 1915 .

p. 361-362. 


\begin{tabular}{|c|c|c|c|c|c|c|}
\hline $\begin{array}{l}\text { Map } \\
\text { number }\end{array}$ & Name & Commodities & $\underline{\text { Size }}$ & Host rock & Comments & $\begin{array}{c}\text { Selected } \\
\text { references } \\
\end{array}$ \\
\hline 1 & $\begin{array}{l}\text { Point Aux } \\
\text { Barques Iighthous }\end{array}$ & e & Occurrence & $\begin{array}{l}\text { Mississippian } \\
\text { Coldwater Formation }\end{array}$ & $\begin{array}{l}\text { Sphalerite and calcite } \\
\text { shell replacement. }\end{array}$ & $\begin{array}{l}\text { Heinrich, 1976, } \\
\text { p. 195; Lane, 1900, } \\
\text { p. 229. }\end{array}$ \\
\hline 2 & $\begin{array}{l}\text { Collison's we } 11, \\
\text { Sne } 11 \text { 's wel1, and } \\
\text { an unnamed well }\end{array}$ & $\mathrm{Pb}$ & do. & Pyritiferous shale & $\begin{array}{l}\text { Galena reported from } \\
3 \text { wells at depths of } \\
40 \text { to } 75 \text { feet. }\end{array}$ & Lane, 1900, p. 161. \\
\hline 3 & Muller's well & $\mathrm{Zn}$ & do. & -- & $\begin{array}{l}\text { Iron and zinc sulfides } \\
\text { reported from a depth of } \\
113-118 \text { feet. }\end{array}$ & Lane 1900, p. 145. \\
\hline 4 & $\begin{array}{l}\text { Bauer's wel1, } \\
\text { Sebewaing }\end{array}$ & $\mathrm{Zn}$ & do. & $\begin{array}{l}\text { Mississippian } \\
\text { Bayport Limestone }\end{array}$ & $\begin{array}{l}\text { Fragments of sphalerite } \\
\text { reported from a depth } \\
118 \text { feet. }\end{array}$ & $\begin{array}{l}\text { Heinrich, 1976, } \\
\text { p. 195; Lane, 1900, } \\
\text { p. 221, 229. }\end{array}$ \\
\hline 5 & Cass City & $\mathrm{Zn}$ & do. & $\begin{array}{l}\text { Mississippian } \\
\text { sandstone (Marshal1 } \\
\text { Formation) }\end{array}$ & $\begin{array}{l}\text { In nodular concretions } \\
\text { and drusy cavities. }\end{array}$ & $\begin{array}{l}\text { Davis, } 1909, \text { p. } 185, \\
\text { 203; He inrich, 1976, } \\
\text { p. 196; Rominger, } \\
\text { 1876, p. 104-105. }\end{array}$ \\
\hline 6 & $\begin{array}{l}\text { Grand Ledge } \\
\text { Clay Products } \\
\text { Company }\end{array}$ & $\mathrm{Zn}, \mathrm{Pb}$ & do. & $\begin{array}{l}\text { Pennsylvanian } \\
\text { Saginaw Formation }\end{array}$ & $\begin{array}{l}\text { In ironstone } \\
\text { concretions. }\end{array}$ & $\begin{array}{l}\text { Dorr and Eschman, } \\
\text { 1970, p. 255; } \\
\text { Heinrich, 1976, } \\
\text { p. 195. }\end{array}$ \\
\hline 7 & Bellevue & $\mathrm{Zn}, \mathrm{Ba}$ & do. & $\begin{array}{l}\text { Mississippian } \\
\text { Bayport Limestone }\end{array}$ & $\begin{array}{l}\text { As fine crystals in } \\
\text { cavities. }\end{array}$ & $\begin{array}{l}\text { Dorr and Eschman, } \\
\text { 1970, p. 255; } \\
\text { Heinrich, 1976, } \\
\text { p. 195, p. } 55 .\end{array}$ \\
\hline 8 & Coldwater & $\mathrm{Zn}, \mathrm{Pb}$ & do. & $\begin{array}{l}\text { Mississippian } \\
\text { Coldwater Shale }\end{array}$ & $\begin{array}{l}\text { In clay-ironstone } \\
\text { concretions. }\end{array}$ & $\begin{array}{l}\text { Dorr and Eschman, } \\
\text { 1970, p. 255; } \\
\text { Heinrich, 1976, } \\
\text { p. } 195 .\end{array}$ \\
\hline
\end{tabular}




\begin{tabular}{|c|c|c|c|c|c|c|}
\hline $\begin{array}{l}\text { Map } \\
\text { number }\end{array}$ & Name & Commodities & $\underline{\text { Size }}$ & Host rock & Comments & $\begin{array}{c}\text { Selected } \\
\text { references } \\
\end{array}$ \\
\hline 1 & $\begin{array}{l}\text { Rossie } \\
\text { (Bigelow, Macomb) }\end{array}$ & $\mathrm{Pb}, \mathrm{Zn}$ & Small & $\begin{array}{l}\text { Precambrian to } \\
\text { Ordovician limestone } \\
\text { and sandstone }\end{array}$ & Silurian mineralization. & $\begin{array}{l}\text { Brown, 1983; } \\
\text { Buddington, 1934; } \\
\text { Neumann, 1952a; } \\
\text { Newland, 1919, } \\
\text { p. 140-142. }\end{array}$ \\
\hline 2 & Redwood & $\mathrm{Pb}$ & do. & $\begin{array}{l}\text { Cambrian sandstone } \\
\text { (Potsdam Sandstone) }\end{array}$ & $\begin{array}{l}\text { Vein; small amount of } \\
\text { galena disseminated in } \\
\text { limestone. }\end{array}$ & $\begin{array}{l}\text { Buddington, 1934, } \\
\text { p. 206-207; Miller, } \\
1910 \text {, p. 58; } \\
\text { Newland, 1919, } \\
\text { p. 142. }\end{array}$ \\
\hline 3 & Pillar Point & $\mathrm{Ba}$ & do. & $\begin{array}{l}\text { Ordovician limestone } \\
\text { (Trenton Limestone) }\end{array}$ & Small veins. & $\begin{array}{l}\text { Merri11, } 1895, \\
\text { p. 582; Newland, } \\
1919 \text {, p. } 33 .\end{array}$ \\
\hline 4 & Martinsburg & $\mathrm{Pb}, \mathrm{Zn}$ & Occurrence & do. & -- & $\begin{array}{l}\text { Miller, 1910, } \\
\text { p. } 58 .\end{array}$ \\
\hline 6 & Salisbury & $\mathrm{Zn}(\mathrm{Pb}, \mathrm{Cu})$ & do. & $\begin{array}{l}\text { Ordovician limestone } \\
\text { (Trenton Limestone) }\end{array}$ & $\begin{array}{l}\text { Filling of solution } \\
\text { cavities and fissures. }\end{array}$ & $\begin{array}{l}\text { Newland, 1919, } \\
\text { p. } 306 .\end{array}$ \\
\hline 7 & Little Falls & $\mathrm{Ba}$ & do. & $\begin{array}{l}\text { Ordovician dolomitic } \\
\text { limestone } \\
\text { (Beekmantown Group?) }\end{array}$ & $\begin{array}{l}\text { In small veins and } \\
\text { geodes. }\end{array}$ & $\begin{array}{l}\text { Newland, } 1919 \text {, } \\
\text { p. } 34 .\end{array}$ \\
\hline 8 & $\begin{array}{l}\text { Saratoga } \\
\text { Springs }\end{array}$ & $\mathrm{Zn}$ & do. & $\begin{array}{l}\text { Ordovician dolomite } \\
\text { (Beekmantown Group) }\end{array}$ & Veins & $\begin{array}{l}\text { Newland, } 1919 \text {, } \\
\text { p. } 306 .\end{array}$ \\
\hline 9 & Blue Corners & $\mathrm{Zn}, \mathrm{Fe}$ & do. & do. & $\begin{array}{l}\text { On small inclined } \\
\text { fractures. }\end{array}$ & $\begin{array}{l}\text { Lesure and Klemic, } \\
1977 .\end{array}$ \\
\hline 10 & White Creek & $\mathrm{Zn}, \mathrm{Pb}$ & Smal1 & $\begin{array}{l}\text { Cambrian quartzite } \\
\text { and minor } \\
\text { conglomerate }\end{array}$ & $\begin{array}{l}\text { Disseminations, semi- } \\
\text { massive lenses, and } \\
\text { minor veinlets (J.F. } \\
\text { Slack, oral commun., } \\
\text { 1987). }\end{array}$ & $\begin{array}{l}\text { Hartnagel and } \\
\text { Broughton, 1951, } \\
\text { p. } 61 \text {; Mather, 1843, } \\
\text { p. 498; New1and, } \\
1919 \text {, p. 143. }\end{array}$ \\
\hline
\end{tabular}


do.

Silurian shale

$\mathrm{Ba}, \mathrm{Sr}, \mathrm{Pb}$

Schoharie

$\mathrm{Zn}$

15 Lockport

do. limestone
Silurian shale and

do. Silurian (?) limestone

do. Silurian dolomite (Lockport Group)

do. Silurian dolomite (Lockport Group) and overlying Holocene peat bogs

Rochester (Penfield and other quarries)

18 Wolcott

19 College Hill

$\mathrm{Zn}(\mathrm{Cu}, \mathrm{Pb})$

20

Buffalo

21
Silver Creek
$\mathrm{Ba}$

$\mathrm{Zn}$

$\mathrm{Ba}$

$\mathrm{Ba}$

n

do.

Silurian dolomite (Lockport Group)

Silurian dolomite (Lockport Group)

do. Devonian

do.

Late Devonian mudrock and shale do. Silurian
Veins $0.25-1.5$

inches wide.

Nodular aggregates of crystals.

Linings of small cavities and stringers.

Metals concentrated in Silurian patch reefs, transported by ground waters and selectively concentrated in peat bogs as finely divided sulfides.

In solution cavities.

In geodes.

Disseminated or in geodes; in reef zones.

"In laminae, in... rounded masses called septaria" (Beck, 1842).

Barite nodules.
Hartnage 1 and Broughton, 1951, p. 61; Newland, 1919 , p. 143.

Beck, 1842, p. 207; Newland, 1919, p. 34 .

Beck, 1842, p. $207-$ 208; Newland, 1919 , p. $34,143$.

Ha 11, 1843, p. 447. New1 and, 1919, p. 307 .

Cannon, 1955.

Bassett and Kinsland, 1973; Jensen, 1942: Kinsland, 1977.

Beck, 1842, p. 209.

Dale, 1953, p. 103-108.

Beck, 1842, p. 209 .

Pepper and others, 1985. 


\begin{tabular}{|c|c|c|c|c|c|c|}
\hline $\begin{array}{l}\text { Map } \\
\text { number }\end{array}$ & Name & Commodities & $\underline{\text { Size }}$ & Host rock & Comments & $\begin{array}{c}\text { Selected } \\
\text { references }\end{array}$ \\
\hline 22 & Laona & $\mathrm{Ba}$ & do. & Devonian & $\begin{array}{l}\text { "In laminae, in... } \\
\text { rounded masses cailed } \\
\text { septaria" (Beck, 1842). }\end{array}$ & $\begin{array}{l}\text { Beck, } 1842, \\
\text { p. } 209 .\end{array}$ \\
\hline 23 & Syracuse & $\mathrm{Ba}$ & do. & $\begin{array}{l}\text { Silurian (?) } \\
\text { limestone }\end{array}$ & $\begin{array}{l}\text { "In plates or tables } \\
\text { interlacing each other" } \\
\text { (Beck, 1842). }\end{array}$ & $\begin{array}{l}\text { Beck, } 1842, \\
\text { p. } 206 .\end{array}$ \\
\hline 24 & $\begin{array}{l}\text { Tully Limestone } \\
\text { ( } 7 \text { localities) }\end{array}$ & $\mathrm{Zn}, \mathrm{Ba}$ & do. & Devonian limestone & $\begin{array}{l}\text { Replacement of matrix } \\
\text { and grains, she } 11 \\
\text { fillings. }\end{array}$ & $\begin{array}{l}\text { Hecke1, 1973, } \\
\text { p. } 59,75, \text { c9. }\end{array}$ \\
\hline 25 & Auburn & $\mathrm{Ba}$ & do. & Devonian? & $\begin{array}{l}\text { "In laminae, in .. } \\
\text { rounded masses cailed } \\
\text { septaria" (Beck, 1842). }\end{array}$ & $\begin{array}{l}\text { Beck, } 1842, \\
\text { p. } 209 .\end{array}$ \\
\hline 26 & $\begin{array}{l}\text { Cayuga and } \\
\text { Seneca Lakes } \\
\text { ( } 3 \text { localities) }\end{array}$ & $\mathrm{Ba}, \mathrm{Zn}$ & do. & $\begin{array}{l}\text { Devonian shale } \\
\text { (Genessee Shale) }\end{array}$ & In limestone concretions. & Martens, 1925. \\
\hline 27 & Canaan & $\mathrm{Pb}, \mathrm{Zn}(\mathrm{Ag})$ & Sma 11 & Limestone & $\begin{array}{l}\text { Veins. Numerous small } \\
\text { veins reported in the } \\
\text { area. }\end{array}$ & $\begin{array}{l}\text { Beck, } 1842, \text { p. } 46 \text {; } \\
\text { Mather, 1843, } \\
\text { p. 500-501. }\end{array}$ \\
\hline 28 & Claverack & $\mathrm{Pb}$ & currence & -- & Vein & $\begin{array}{l}\text { Mather, 1843, } \\
\text { p. 502; Beck, 1842, } \\
\text { p. } 46 .\end{array}$ \\
\hline 29 & Livingston & $\mathrm{Pb}, \mathrm{Zn} ?$ & do. & -- & $\begin{array}{l}\text { In an old mine in } \\
\text { Livingston. }\end{array}$ & $\begin{array}{l}\text { Mather, } 1843 \text {, } \\
\text { p. 503. }\end{array}$ \\
\hline 30 & $\begin{array}{l}\text { Ancram } \\
\text { (Livingston } \\
\text { lead mine) }\end{array}$ & $\mathrm{Pb}(\mathrm{Cu}, \mathrm{Zn}, \mathrm{Ba}, \mathrm{Ag})$ & Sma11 & $\begin{array}{l}\text { Ordovician dolomite } \\
\text { (Wallomsac } \\
\text { Formation) }\end{array}$ & $\begin{array}{l}\text { Mineralization in faults } \\
\text { of possible Triassic age } \\
\text { N.M. Radcliff, oral } \\
\text { commun., 1981). }\end{array}$ & $\begin{array}{l}\text { Hartnage } 1 \text { and } \\
\text { Broughton, 1951, } \\
\text { p. 61; Beck, 1842, } \\
\text { p. 45-46, 409; } \\
\text { Mather, 1843, } \\
\text { p. 498-500; Newl and, } \\
1919, \text { p. 142-143; } \\
\text { Peterson, 1950, } \\
\text { p. 47. }\end{array}$ \\
\hline
\end{tabular}


Map
number

31

32

Fishkill

33

Shawangunk

district

(Guymard,

Ellenville,

Shawangunk,

Ulster and

Otisville

mines)

Haverstraw
$\mathrm{Pb}(\mathrm{Cu}, \mathrm{Ag})$

$$
\text { do. }
$$

Ordovician limestone

Many smal1 veins.

In a thin vein of quartz.

In sheeted zones along fau 1ts; veins and stockworks. and conglomerate

(Shawangunk

Cong lomerate)

Beck, 1842, p. 46; Mather, 1843,

p. 500-502; Newland, 1919, p. 143.

Mather, 1843, p. 503.

Crawford and Beales, 1983, p. 436-445; Eilertsen, 1950;

Gray, 1961; Ingham, 1940; Mather, 1843, p. 359-362; Newland, 1919, p. 300-306; Neumann, 1952b; Sims and Holtz, 1951.

Minute crystals associated with calcite.

Beck, 1842, p. 206. 


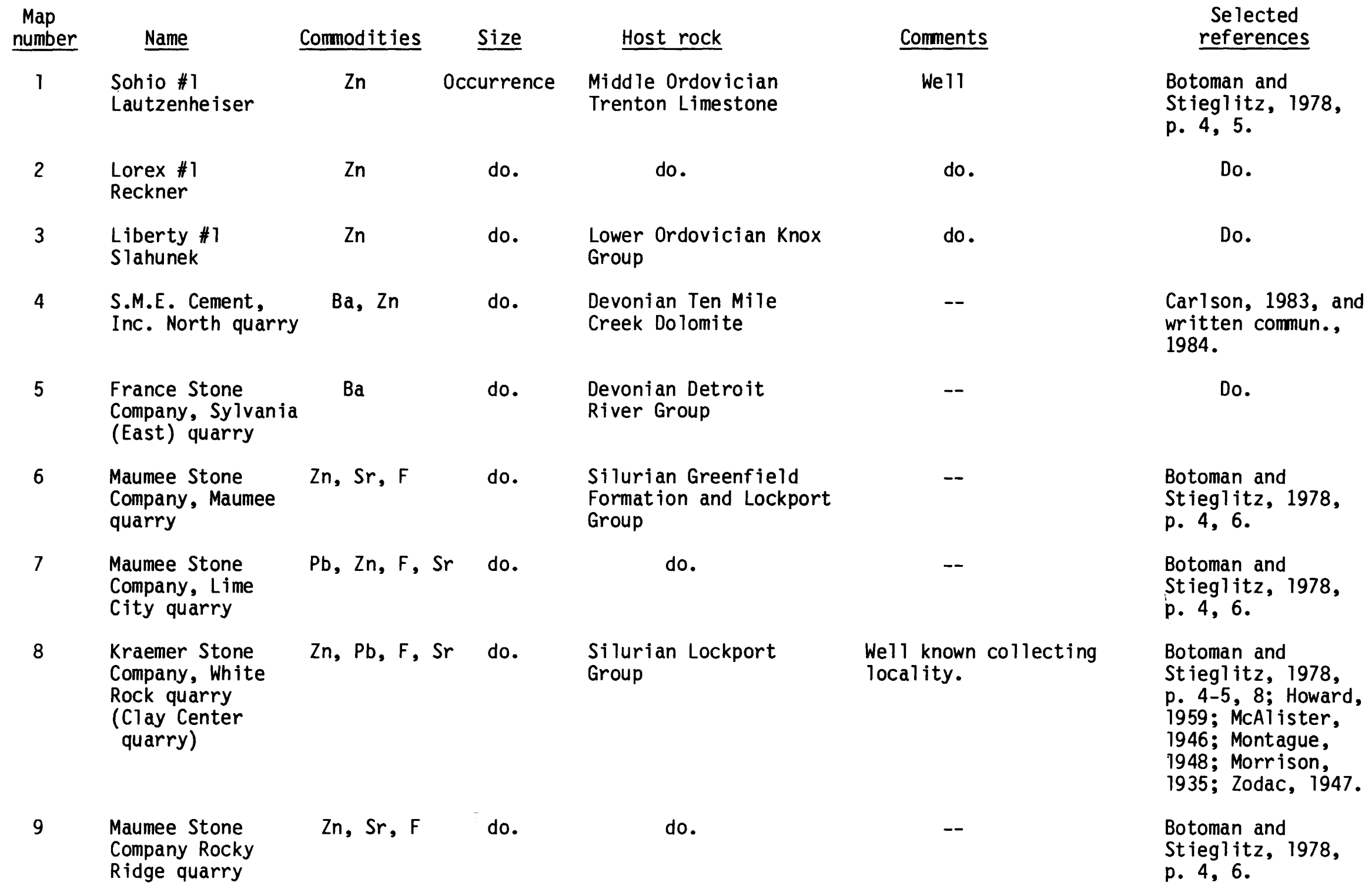


Map

number

10

01d Purtee

quarry

11

U.S. Gypsum
Company,
Genoa quarry

12

Ohio Lime

Company Woodville $\mathrm{Zn}, \mathrm{Pb}, \mathrm{Ba}$

quarry and

Martin Marietta

Chemicals

quarries

Charles Pfizer and Company

quarry

Kellstone (North side) quarry

Kellstone (South

side) quarry

16

Sandusky Crushed

Stone Company

quarry

Leeds' barite

occurrence

18

Milan
$\mathrm{Zn}, \mathrm{Pb}, \mathrm{F}, \mathrm{Sr}$

$\mathrm{Zn}$

Zn

Zn

$\mathrm{Ba}$

Zn

$\mathrm{Zn}, \mathrm{Pb}, \mathrm{F}$

do.

o.

do.

do.

do.

o.

Devonian Lucas

Dolomite and

Columbus Limestone

do.

do.

do.

Devonian Detroit

River Group

do.

$\mathrm{Ba}, \mathrm{Zn}$
Devonian Huron

Shale

Devonian Huron

Member of the Ohio

Shale
Selected

references

Carlson, 1983, and written commun., 1984.

Botoman and

Stieglitz, 1978,

p. 4, 6;

E. H. Carlson, 1984, written commun.

Botoman and

Stieglitz, 1978,

p. 4, 6; Green, 1971 .

Botoman and Stieglitz, 1978; Carlson, 1983, and written commun., 1984 .

Carlson, 1983, and written commun., 1984.

Do.

Do.

Coal seams traversed by Leeds, 1875, p. 105. "sheets" of barite.

In concretions.

Hyde and Landy, 1966; Leavens, 1968. 
Map

number

19

Vermilion

River

Commodities

$\underline{\text { Size }}$

Host rock

do. Devonian Cleveland

Member of the Ohio Shale

20

Maumee Stone

Company,

Auglaize

quarry

21

France Stone

Company

(formerly Pugh)

quarry

22

France Stone $\mathrm{Zn}, \mathrm{F}$

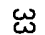

Company North

Baltimore

quarry

MacRitchie

Material, Inc.

quarry

24 Basic Refractories

Combustion

Engineering quarry

0ld quarr

26

Ottawa Stone

Company, Inc. quarry

27

Putnum Stone

Company, Inc.

quarry

28

Stone quarry
$\mathrm{Zn}, \mathrm{F}$

$\mathrm{Zn}, \mathrm{Ba}, \mathrm{Sr}$

do.

do.

Detroit River

Formations

$$
\text { o. }
$$

do.

Silurian Tymochtee

Formation

do. Silurian Greenfield

Formation and Lockport

Group

do.

do.

do.

do. Silurian Raisin River

and Tymochtee

Formations

do.

do. Silurian Tymochtee

Formation
Comments

Selected

references

In concretions. 1979.

\section{Botoman and}

Stieglitz, 1978 ,

p. 4,6 ; E. H.

Carlson, 1984,

written commun.

Botoman and

Stieglitz, 1978,

p. 4, 6; Parr and

Chang, 1977, 1978 ,

$1979,1980$.

Botoman and

Stieglitz, 1978 ,

p. 4,6 .

Carlson, 1983, and written commun.

1984.

Do.

Do.

Botoman and

Stieglitz, 1978,

p. 4,6 .

Do.

Do. 
Map

number

29 Delphos Quarries
Company quarry

Commodities

$\mathrm{Zn}, \mathrm{F}$

Nationa 1 Lime

and Stone Company,

Rimer quarry

J. W. Karch Stone

Company quarry

$\mathrm{Zn}$

Zn

32

National Lime

and Stone Company,

Buckland quarry

National Lime

and Stone Company,

Lima quarry

Western Ohio

Stone Company

quarry

Nationa 1 Lime and

Stone Company,

Findlay quarry

\section{Ashland \#1}

Cotner

$\mathrm{Zn}$

$\mathrm{Zn}, \mathrm{F}$

,

$\mathrm{Zn}$

Zn

$\mathrm{Zn}$

National Lime $\quad \mathrm{Zn}, \mathrm{Sr}$

and Stone

Company, Carey

quarry

Brinkerhoff \#1

$\mathrm{Zn}$

Fox
White \#1

Stubb

\section{Size}

Host rock

Comments

do. Silurian Tymochtee Formation

do. Silurian Raisin River and Tymochtee Formations

do. Silurian Greenfield

Formation

do. Silurian Greenfield Formation and Lockport Group

do. Silurian Tymochtee Formation

do.

$$
\text { do. }
$$

do. Silurian Tymochtee Formation

do. Middle Ordovician Wells Creek (Glenwood) Formation

do. Silurian Greenfield

Formation and Lockport Group

do. Lower Ordovician Knox Dolomite

do. Middle Ordovician Trenton Limestone
We 11

Do.

Do.

Carlson, 1983, and written commun. 1984.

Botoman and Stieglitz, 1978, p. 4,5 .

Do.

Do.

Carlson, 1983 and written commun., 1984.

Botoman and Stieglitz, 1978, p. 4,5 .

Botoman and Stieglitz, 1978, p. 4,6 .

We 11

Do.

Well
Botoman and Stieglitz, 1978, p. 4,5 . 
Silurian Raisin River and Tymochtee Formations

41 Hardin Quarry Zn, F Company quarry

McMahon-Bullington \# 1 Wolf

43

Wooster, outcrop $\mathrm{Zn}(\mathrm{Pb})$

near

44

Blood \#

Sch labach,

Blood \#2

Schmidt

Marshallville, outcrops near

Coal mine

Zn

$\begin{array}{ll}\text { Ellsworth, } & \mathrm{Zn}, \mathrm{Ba} \\ \text { outcrop near }\end{array}$

outcrop near

$\mathrm{Zn}, \mathrm{Ba}(\mathrm{Pb})$

do.

Pennsylvanian shale of the basal Pottsville Formation above unconformity

do.

Pennsylvanian

Al legheny Group

do.

Pennsylvanian

do.

Pennsylvanian
Al legheny Group

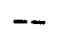

$-$

We 11

\section{references}

Botoman and

Stieglitz, 1978, p. 4,6 .

Botoman and

Stieglitz, 1978, p. 4,5 .

Do.

As crystals in shale, as fillings or replacements of fossils, and as fossiliferous

concretions associated with pyrite and calcite.

Wells; rare galena as a vug filling.

In clay-ironstone septarian concretions in black, bituminous, thinly laminated shale.

Wurtzite in concretions in coal.

In shrinkage cracks

in siderite

in shale.

Wurtzite in siderite concretions in shale.
Botoman and

Stieglitz, 1978 p. 4, 6; Ver Steeg, 1940 .

Botoman and p. 4, 6; Multer, 1963, p. 13.

Botoman and p. 4, 6; Ver Steeg, 1942 .

Botoman and Stieglitz, 1978, p. 4, 6; Seaman and Hamilton, 1950.

Botoman and Stieglitz, 1978 , p. 4, 6; Greene, 1935.

Hollenbaugh and Carlson, 1983.
Stieglitz, 1978, Stieglitz, 1978 , 
do.

do.

do.

do.

do.

do.

do.

do.

do.

do.

$53 \quad$ Site 6

Site 7

Steubenville

do.
Pennsylvanian Conemaugh Group

\section{do. Silurian Brassfield Formation}

Survey strati-

graphic section 12771

56

Ohio Geologica

Survey strati-

graphic section

15756, 01d Marble

Cliff, Lewisburg

quarry

57

Ohio Geological

Survey strati-

graphic section

15772

58 Cominco-American

CA-54 Swingley

do.

do.

Dolomite

do. Silurian Greenfield

Formation do.

Do.

do.

Do.

do.

Do.

do.

do.

do.

$-$

We 11

Lower Ordovician Knox
Do.

Do.

Botoman and

Stieglitz, 1978, p. 4, 6; Hollenbaugh and Carlson, 1983;

Seaman and Hamilton, 1950.

Botoman and

Stieglitz, 1978,

p. 4,5 .

Do.

Do.

Botoman and Stieglitz, 1978, p. 4,6 .

Botoman and Stieglitz, 1978, p. 4, 5; Rogers, 1936, p. 114. 
Map

number

Name

Commodities

60

Blue Rock quarry, Zn

American

Aggregates

Corporation

61

01d Rucker quarry

$\mathrm{Zn}$

62

Ohio Geologica

Survey

stratigraphic

section 13610;

Davon Inc.,

Highland Plant

quarry

63 Marshall quarry

64 Ohio Geological

Survey

stratigraphic

section 13609

65

Serpent Mound

\section{$\underline{\text { Size }}$}

do.

do. Silurian Brassfield
Zn

Zn

$\mathrm{Zn}, \mathrm{Ba}$

$\underset{\mathrm{Ba}}{\mathrm{Zn}}, \mathrm{F}$,

do.

do.

do.
Host rock

Silurian Greenfield

Formation

do.

Formation

\section{Comments}

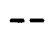

$-$

$--$

Silurian Lilley-Peebles Formation

Silurian Brassfield Formation

Silurian dolomite. Greenfield LilleyPeebles, Bisher, and Brassfield Formations structure.
Selected

references

Botoman and

Stieglitz, 1978 ,

p. 4,5 .

Botoman and

Stieglitz, 1978,

p. 4, 6; Napper, 1917 .

Botoman and Stieglitz, 1978 , p. 4,5 .

Do.

Botoman and Stieglitz, 1978, p. 4,5 .

In shatter breccia in cryptoexplosion
Botoman and

Stieg 1 itz, 1978 ,

p. 4, 5; A. V. Hey 1

1983, oral commun.;

Heyl and Brock.

1962; Reidel, 1975;

Reidel and Koucky,

1981; Stout, 1941,

p. 17,78 . 


\begin{tabular}{|c|c|c|}
\hline $\begin{array}{l}\text { Map } \\
\text { number }\end{array}$ & Name & Commodities \\
\hline 66 & $\begin{array}{l}\text { Ohio Geological } \\
\text { Survey } \\
\text { stratigraphic } \\
\text { section } 14350\end{array}$ & $\mathrm{Zn}$ \\
\hline 67 & $\begin{array}{l}\text { Ohio Geological } \\
\text { Survey } \\
\text { stratigraphic } \\
\text { section } 9732\end{array}$ & $\mathrm{Zn}$ \\
\hline 68 & $\begin{array}{l}\text { Cominco-American } \\
\text { \#1 Rockey }\end{array}$ & $\mathrm{Zn}, \mathrm{Ba}$ \\
\hline 69 & $\begin{array}{l}\text { Ohio Geological } \\
\text { Survey } \\
\text { stratigraphic } \\
\text { section } 12667\end{array}$ & $\mathrm{Zn}, \mathrm{Ba}$ \\
\hline 70 & $\begin{array}{l}\text { Cominco-American } \\
\text { \#1 Hughes }\end{array}$ & $\begin{array}{l}\mathrm{Zn}, \mathrm{Ba}, \\
\mathrm{Cu} ?)\end{array}$ \\
\hline 71 & $\begin{array}{l}\text { 01d Glouster } \\
\text { Brick quarry }\end{array}$ & $\mathrm{Zn}$ \\
\hline
\end{tabular}

\section{Size \\ do. \\ Host rock \\ Silurian Brassfield Formation}

do. Silurian Brassfield

Formation

do.

Lower Ordovician Knox

do. Group

Silurian Brassfield

Formation
$\mathrm{Zn}, \mathrm{Ba}$,
$\mathrm{Cu} ?)$

do.

do.
Lower Ordovician Knox Dolomite

Pennsylvanian Conemaugh Group

\section{Comments}

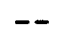

We 11

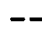

We 11

Wurtzite in siderite concretions in shale.
Selected

references

Botoman and Stieglitz, 1978, p. 4,5 .

Do.

Do.

Do.

Do.

Botoman and Stieglitz, 1978, p. 4, 6; Seaman and Hami iton, 1950. 


\section{PENNSYLVANIA}

\begin{tabular}{|c|c|c|c|c|c|c|}
\hline $\begin{array}{l}\text { Map } \\
\text { number }\end{array}$ & $\underline{\text { Name }}$ & Commodities & $\underline{\text { Size }}$ & Host rock & Comments & $\begin{array}{l}\text { Selected } \\
\text { references }\end{array}$ \\
\hline 1 & $\begin{array}{l}\text { Harrison } \\
\text { Township }\end{array}$ & $\mathrm{Zn}(?), \mathrm{Pb}(?)$ & Occurrence & -- & -- & $\begin{array}{l}\text { Smith, } 1977, \\
\text { p. } 295 .\end{array}$ \\
\hline 2 & $\begin{array}{l}\text { Ridgebury, } \\
\text { east of } \\
\text { ( } 2 \text { localities) }\end{array}$ & $\mathrm{Pb}, \mathrm{Cu}$ & Occurrences & $\begin{array}{l}\text { Devonian Catskill } \\
\text { Formation }\end{array}$ & -- & $\begin{array}{l}\text { Smith, } 1977, \\
\text { p. } 289 .\end{array}$ \\
\hline 3 & $\begin{array}{l}\text { Roaring Branch } \\
\text { of Lycoming } \\
\text { Creek }\end{array}$ & $\mathrm{Pb}, \mathrm{Zn}, \mathrm{Cu}$ & Occurrence & $\begin{array}{l}\text { Upper Devonian } \\
\text { Catskill Formation }\end{array}$ & -- & $\begin{array}{l}\text { Smith, } 1977, \\
\text { p. } 293 .\end{array}$ \\
\hline 4 & $\begin{array}{l}\text { Millview } \\
\text { Quarry }\end{array}$ & $\begin{array}{l}\mathrm{Pb}, \mathrm{Zn} \\
(\mathrm{As}, \mathrm{Cu})\end{array}$ & do. & $\begin{array}{l}\text { Devonian coquinoid } \\
\text { limestone (Catskill } \\
\text { Formation) }\end{array}$ & $\begin{array}{l}\text { Disseminated grains, } \\
\text { blebs and veinlets; } \\
\text { mostly concentrated } \\
\text { near top of coquinite } \\
\text { bed. }\end{array}$ & $\begin{array}{l}\text { Smith, } 1977, \\
\text { p. 221-222. }\end{array}$ \\
\hline 5 & Prospect 12 & $\mathrm{~Pb}, \mathrm{Cu}$ & do. & $\begin{array}{l}\text { Devonian sandstone } \\
\text { (Catskill Formation) }\end{array}$ & -- & $\begin{array}{l}\text { Smith, } 1977, \\
\text { p. } 291 .\end{array}$ \\
\hline 6 & $\begin{array}{l}0 \text { il City, } \\
\text { east of }\end{array}$ & $\mathrm{Pb}, \mathrm{Zn}$ & do. & -- & 0il-well cutting. & $\begin{array}{l}\text { Smith, } 1977, \\
\text { p. } 295 .\end{array}$ \\
\hline 7 & $\begin{array}{l}\text { Sugar Hill } \\
\text { Quarry }\end{array}$ & $\mathrm{Zn}, \mathrm{Ba}(\mathrm{Cu})$ & do. & $\begin{array}{l}\text { Pennsylvanian } \\
\text { siderite nodules in } \\
\text { shale above the } \\
\text { Vanport Limestone }\end{array}$ & $\begin{array}{l}\text { Sphalerite and wurtzite } \\
\text { in nodules. }\end{array}$ & $\begin{array}{l}\text { Smith, } 1977, \\
\text { p. } 223-225 .\end{array}$ \\
\hline 8 & $\begin{array}{l}\text { Coal mine } \\
\text { west of Parker }\end{array}$ & $\mathrm{Pb}$ & do. & $\begin{array}{l}\text { Carboniferous fire } \\
\text { clay }\end{array}$ & -- & $\begin{array}{l}\text { Smith, } 1977 \text {, } \\
\text { p. } 289 .\end{array}$ \\
\hline 9 & $\begin{array}{l}\text { Raccoon Creek } \\
\text { near Beaver }\end{array}$ & $\mathrm{Pb}, \mathrm{Zn}$ & do. & Coal measures & Nodules & $\begin{array}{l}\text { Smith, } 1977, \\
\text { p. } 288 .\end{array}$ \\
\hline 10 & $\begin{array}{l}\text { North } \\
\text { Vandergrift }\end{array}$ & $\mathrm{Zn}, \mathrm{Pb}$ (?) & do. & $\begin{array}{l}\text { Limy shale at base } \\
\text { of Upper Freeport } \\
\text { coal }\end{array}$ & Nodules & Do. \\
\hline
\end{tabular}


$\mathrm{Zn}, \mathrm{Ba}, \mathrm{Cu}$ Occurrences
11 Valley Camp, brickyard quarry

G.lassmere Brick Company quarry

Wittmer

Donohoe

$$
\text { Victor (Hector) }
$$

$\mathrm{Pb}, \mathrm{Zn}$ Hollow

\}

$\begin{array}{llc}16 & \text { Scranton } & \mathrm{Zn} \\ 17 & \begin{array}{l}\text { Sul1ivan Trail } \\ \text { Coal Company, } \\ \text { west }\end{array} & \mathrm{Zn} \\ 18 & \begin{array}{l}\text { Pine Hill, } \\ \text { Milford }\end{array} & \mathrm{Zn} \\ 19 & \begin{array}{l}\text { Milford Road } \\ 20\end{array} & \mathrm{Zn}(?)(\mathrm{Pb}, \mathrm{Cu}) \\ 21 & \begin{array}{l}\text { DeHass, A. M. } \\ \text { Lymehurst } \\ \text { quarry }\end{array} & \mathrm{Pb} \\ \end{array}$

Pennsylvanian black shales (Conemaugh Formation)

Occurrence

Mississippian Pocono Sandstone

\section{do.}

do.

do. Devonian Mahantango Siltstone

do. Devonian Hamilton Sand stone

do. Silurian Tonoloway Limestone (?)

do. Silurian 1 imestone (Tonoloway Formation)
The four most productive mineral localities for occurrence of wurtzite along shrinkage cracks in clay-ironstone concretions. Eleven other occurrences listed by Seaman and Hami iton (1950).

Considered by Smith (1977) Smith, 1977, p. 291. to have a reasonable but

slight chance of indicat-

ing a significant prospect

in the area.

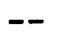

Pennsylvanian

do.
Smith, 1977, p. 292.

Smith, 1977, p. 293.

Smith, 1977, p. 294.

Do.

Smith, 1977, p. 291.

In a calcite veinlet.
Lapham and Geyer 1959, p. 29-30; Seaman and Hamilton, 1950 , p. 43-50. 


\begin{tabular}{|c|c|c|c|c|c|c|}
\hline $\begin{array}{l}\text { Map } \\
\text { number }\end{array}$ & Name & Commodities & $\underline{\text { Size }}$ & Host rock & Comments & $\begin{array}{c}\text { Selected } \\
\text { references }\end{array}$ \\
\hline 11 & $\begin{array}{l}\text { Valley Camp, } \\
\text { brickyard } \\
\text { quarry }\end{array}$ & & & & & \\
\hline 12 & $\begin{array}{l}\text { Glassmere Brick } \\
\text { Company quarry }\end{array}$ & & & & & \\
\hline 13 & Wittmer & $\mathrm{Zn}, \mathrm{Ba}, \mathrm{Cu}$ & Occurrences & $\begin{array}{l}\text { Pennsylvanian black } \\
\text { shales (Conemaugh } \\
\text { Formation) }\end{array}$ & $\begin{array}{l}\text { The four most productive } \\
\text { mineral localities } \\
\text { for occurrence of } \\
\text { wurtzite along shrinkage } \\
\text { cracks in clay-ironstone } \\
\text { concretions. Eleven other } \\
\text { occurrences listed by } \\
\text { Seaman and Hamilton (1950). }\end{array}$ & $\begin{array}{l}\text { Lapham and Geyer } \\
1959 \text {, p. } 29-30 ; \\
\text { Seaman and Hamilton, } \\
1950 \text {, p. } 43-50 \text {. }\end{array}$ \\
\hline 14 & Donohoe & & & & & \\
\hline 15 & $\begin{array}{l}\text { Victor (Hector) } \\
\text { Hollow }\end{array}$ & $\mathrm{Pb}, \mathrm{Zn}$ & Occurrence & $\begin{array}{l}\text { Mississippian } \\
\text { Pocono Sandstone }\end{array}$ & $\begin{array}{l}\text { Considered by Smith ( } 1977) \\
\text { to have a reasonable but } \\
\text { slight chance of indicat- } \\
\text { ing a significant prospect } \\
\text { in the area. }\end{array}$ & Smith, 1977, p. 291. \\
\hline 16 & Scranton & $\mathrm{Zn}$ & do. & $\begin{array}{l}\text { Pennsylvanian } \\
\text { coal measures }\end{array}$ & -- & Smith, 1977, p. 292. \\
\hline 17 & $\begin{array}{l}\text { Sullivan Trail } \\
\text { Coal Company, } \\
\text { west }\end{array}$ & $\mathrm{Zn}$ & do. & do. & & Smith, 1977, p. 293. \\
\hline 18 & $\begin{array}{l}\text { Pine Hill, } \\
\text { Milford }\end{array}$ & $\mathrm{Zn}$ & do. & $\begin{array}{l}\text { Devonian Mahantango } \\
\text { Siltstone }\end{array}$ & -- & Smith, 1977, p. 294. \\
\hline 19 & Milford Road & $\mathrm{Zn}(?)(\mathrm{Pb}, \mathrm{Cu})$ & do. & $\begin{array}{l}\text { Devonian Hamilton } \\
\text { Sandstone }\end{array}$ & -- & Do. \\
\hline 20 & DeHass, A. M. & $\mathrm{Pb}$ & do. & $\begin{array}{l}\text { Silurian Tonoloway } \\
\text { Limestone (?) }\end{array}$ & -- & Smith, 1977, p. 291. \\
\hline 21 & $\begin{array}{l}\text { Lymehurst } \\
\text { quarry }\end{array}$ & $\mathrm{Pb}, \mathrm{Zn}$ & do. & $\begin{array}{l}\text { Silurian limestone } \\
\text { (Tonoloway Formation) }\end{array}$ & In a calcite veinlet. & $\begin{array}{l}\text { Smith, } 1977, \\
\text { p. } 180-182 .\end{array}$ \\
\hline
\end{tabular}


Zn quarry do.
$\mathrm{Pb}, \mathrm{Cu}, \mathrm{As})$

Limestone, upper Late Silurian age and possibly lowermost

Keyser Formation of Devonian age.

Almedia mine

area (Webb

mine, Dave's

Seafood outcrops)

Buffalo Valley

$\mathrm{Pb}$

N

$\mathrm{Pb}, \mathrm{Zn}$

( $\mathrm{Cu}, \mathrm{As}, \mathrm{Ag})$

do.

do.

Devonian mudstone (Marcellus Formation)

do.

do.

Silurian Tonoloway(?)

Limestone
Winfield quarry

$\mathrm{Zn}, \mathrm{Pb}$

do.

Sma11

(Cu)

(Dougherty)

28

Kreamer

29

Penn Haven Junction

Silurian limestone (upper part of the Tonoloway Formation)

Silurian 1 imestone (Tonoloway Formation)

do.

Ridgley Member, old Port Formation below Needmore shale)

do. Devonian sandstone (Catskill Formation) Tonoloway Formation of

In calcite veins. references

Smith, 1977. p. 177-180.

In nodules.

Veins in outcrop, ore in a fracture zone, as irregu lar replacements of limestone and along joint planes. Possibly

a crackle breccia.

Replacement of pyritebearing laminated limestone; calcite and sphalerite gash veins.

In calcite veins and a replacement bed.

Occurrence of clausthalite and uraninite on quartzencrusted joint surfaces and dissemiriated.
Way and Smith, 1983.

Smith, 1977, p. 163-168.

Smith, 1977 p. 295.

Smith, 1977 , p. 185-187.

Smith, 1977 , p. $170-175$.

Smith, 1977, p. 295.

Klemic and others, 1963; p. 67, 78-79, 87: Smith, 1977 , p. 290 .

Smith, 1977, p. 290. 


\section{Commodities}

Zn

Friedensville District-New

Ueberroth mine,

01d Hartman

mine, Correll or

Saucon mine,

Triangle or

Three Corners

mine

Included Greene mine in 42

$\mathrm{Zn}$

Included

Al lentown quarry at Little LehighJordan Creek

43

Rickenback

$\mathrm{Zn}$

44

0ley Valley

$\mathrm{Zn}(\mathrm{Cu})$

Frystown

$\mathrm{Ba}$

Mi lesburg Gap

area

$47 \quad$ Skytop

\section{Size}

Large

do.

do.

45

46

$\mathrm{Ba}$

$\mathrm{Zn}, \mathrm{Pb}, \mathrm{Ba}$

$\mathrm{Pb}, \mathrm{Ba}$ Hartman mine,

Host rock

Lower Ordovician

dolomite (Rickenback

town Group)

Occurrence

Jacksonburg Formation
and (or) Beekmantown Group

Cambrian Limestone

(Allentown Formation)

Lower Ordovician

Beekmantown Group

Prospects

Lower and Middle

Ordovician dolomite and limestone

(Ontelaunee Formation)
Prospects

\section{Cambro-Ordovician \\ shale (Hamburg}

sequence)

Prospects
Silurian quartzite
(Tuscarora
Formation)

Occurrence

Silurian quartzite
Formation of Beekman-

Comments

Replacement of breccias.

Callahan, 1968 , p. 94-107; Rose, 1970, p. 7-8;

Smith, 1977 , p. $82-88,102-111$, 140-149.

Considered by Smith

(1977) to have a

reasonable but slight chance of indicating

a significant prospect

in the area.

Do.

Do.

In breccias near the

crest of an anticline.

Fetid barite nodule-

like fragments.

In veinlets, along

faults, in breccias.

(Tuscarora and Juniata

Formations)
Smith, 1977

p. 29 .
Do.

Smith, 1977 , p. 28.

Smith, 1977 , p. $112-113$.

Smith, 1974, p. 4-7; Berkheiser, 1984 ,

$43 \mathrm{p}$.

Smith, 1977, p. 208, 217.

Smith, 1977, p. 209. 
Keystone mine area, North Sinking Valley (approximately 30 localities)

\section{Southern}

Sinking Valley area, including Albright farm occurrence (14 localities)

Canoe Creek quarry

Thompson

Hares Va1ley area (14 locations)

Occurrence

do.

Zn

$\mathrm{Pb}, \mathrm{Zn}$

Prospect

$\mathrm{Zn}, \mathrm{Pb}, \mathrm{As}$

Prospects and occurrences limestone and

dolomite

Ordovician limestone and dolomite a stratigraphic zone. disseminated.
Cambrian through

Middle Ordovician

In veins confined to

In veins and

Silurian 1 imestone

$\mathrm{Zn}(\mathrm{Pb})$

Occurrences

do. Sproul Lime and Stone Company Quarries
Soister Limonite $\mathrm{Fe}(\mathrm{Zn})$ Mine (3 locations)
$\mathrm{Zn}$ Ordovician dolomite
(Beekmantown Group)

\section{Devonian sandstone} (Chemung(?)

Formation) (Keyser Formation)

Silurian limestone

(Tonoloway Formation)

Silurian clastic rocks (mainly Tuscarora Formation)

Ordovician dolomite (Bellefonte and upper Axemann Formations)

\section{Galena disseminated}

in sandstone.

Mineralization on a joint.

Mineralization along and near a joint.

Mineralization along joints, associated with faults, thin veins,

1 imonite gossans.

In dolomite bed that has Smith, 1977, p. 115been partly replaced by 120 . silica, in chert nodules, quartz and dolomite

rosettes and in shaly

laminae between beds.

In calcite-filled joints. Smith, 1977, p. 176Silurian-Devonian limestone (Tonoloway
Possibly in a collapserubble breccia.
Rose, 1970, p. 8; Smith, 1977, p. 89102.

Rose, 1970 , p. 8; Smith, 1977 , p. 124 140 .

Smith, 1977, p. 219.

Smith, 1977 , p. 168 170.

Smith, 1977 , p. $183-$ 185.

Smith, 1977, p. 188207; Smith and others, 1971. 177.

mith, 1977, p. 120124. 


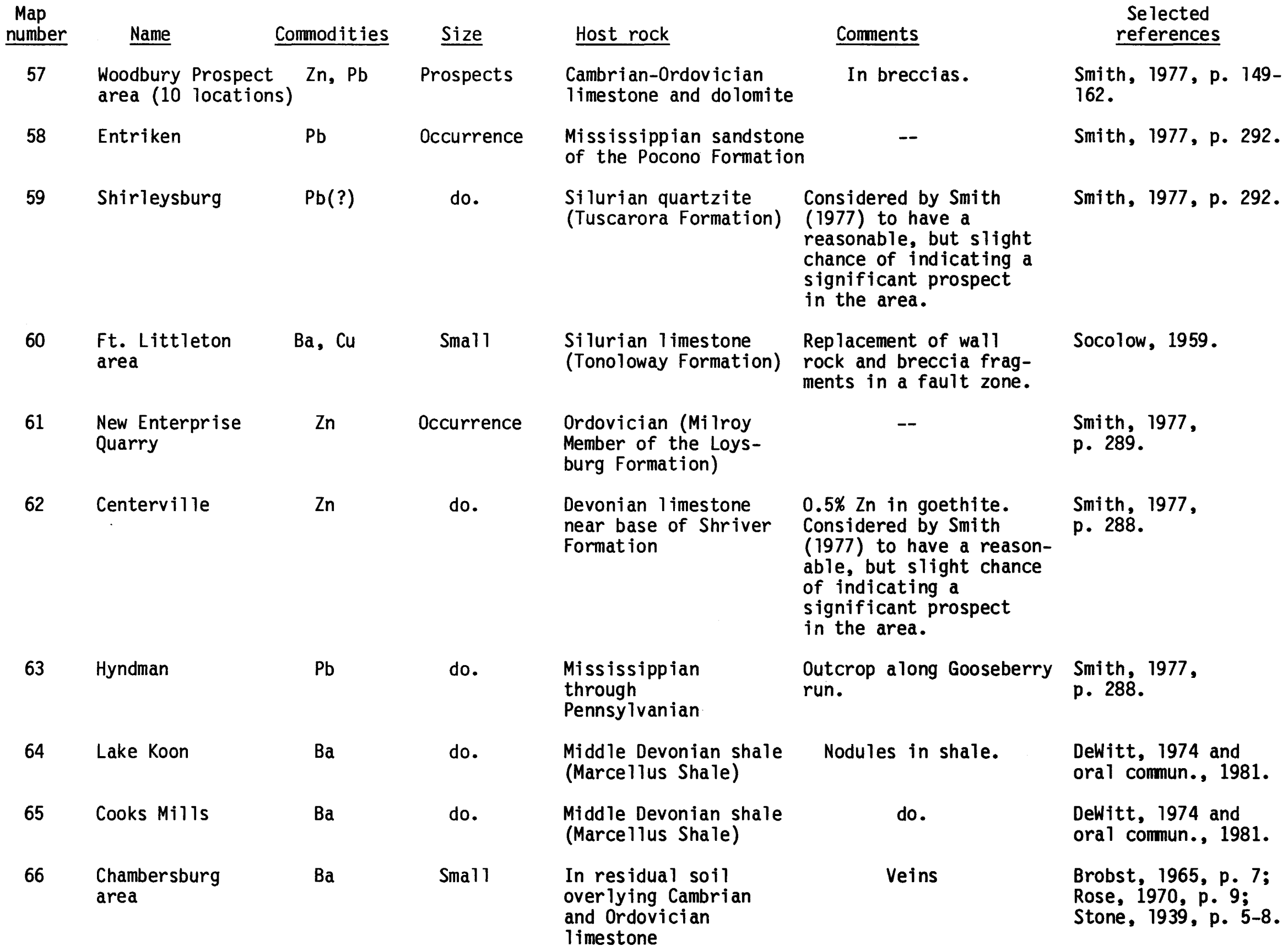




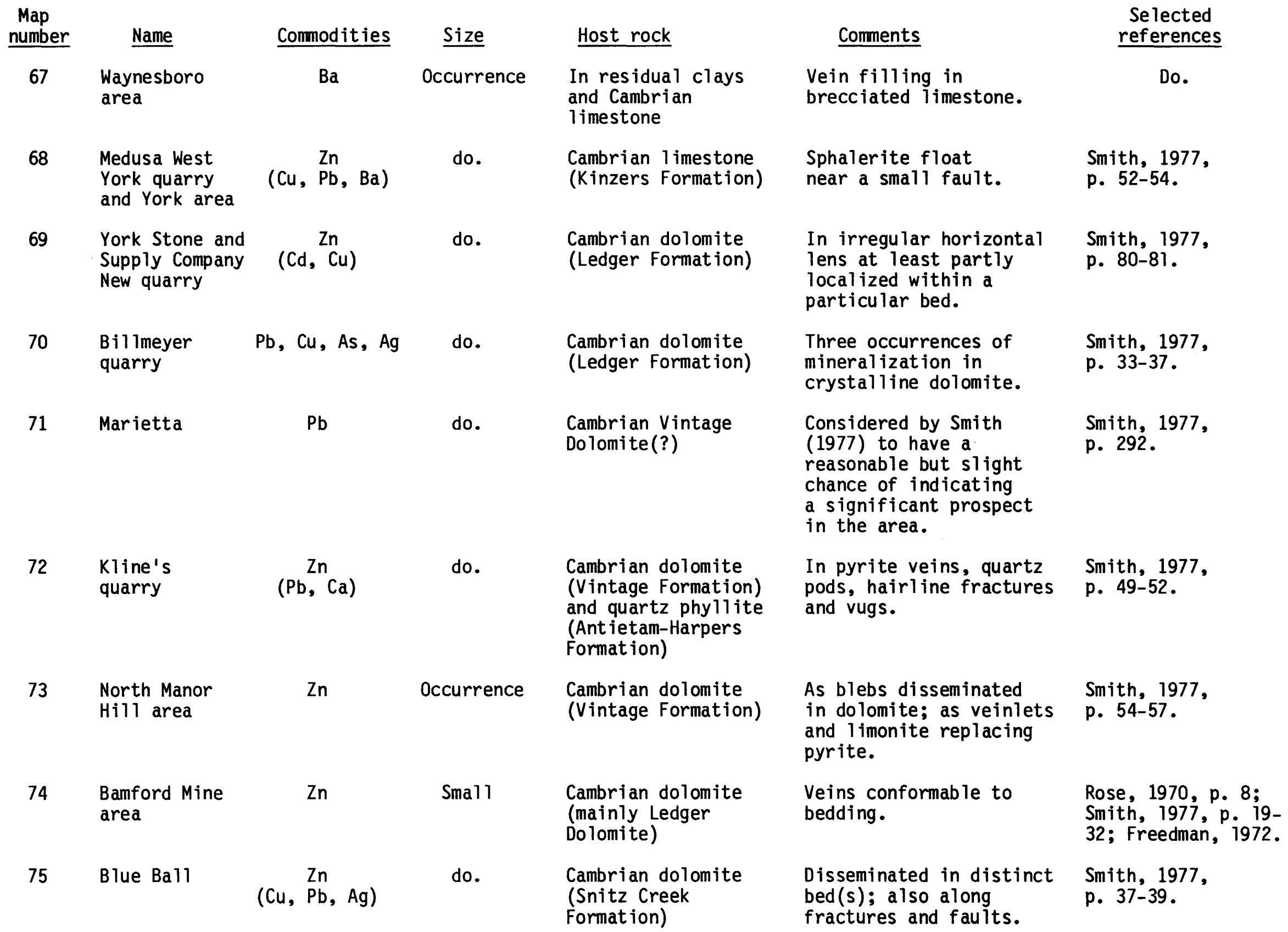


Map

number

Name

76

Gap Northeast

77 Pequea and

Burnt Mills

Silver Mines

Area
Host rock

do.

$\mathrm{Zn}$
$(\mathrm{Pb}, \mathrm{Cu})$

Prospect

$\mathrm{Pb}, \mathrm{Ag}$

Sma 11

Cambrian dolomite

(Vintage Formation)

\section{Comments}

Geochemical anomalies for zinc to the south and southwest.

In quartz veins along bedding planes.

Smith, 1977 , p. 46-49.

Rose, 1970, p. 8; Smith, 1977

p. 62-79. 


\begin{tabular}{|c|c|c|}
\hline $\begin{array}{c}\text { Map } \\
\text { number }\end{array}$ & Name & Commodities \\
\hline 1 & $\begin{array}{l}\text { J. Curt is No. } 1 \\
\text { Memph is Equ ipment } \\
\text { Company }\end{array}$ & $\mathrm{Zn}$ \\
\hline 2 & $\begin{array}{l}\text { Heywood Cordle } \\
\text { No. } 1 \text {, Memphis } \\
\text { Equipment Company }\end{array}$ & $\mathrm{Zn}$ \\
\hline 3 & $\begin{array}{l}\text { Fee (Joyner) } \\
\text { No. 1, } \\
\text { Draughon Bros. }\end{array}$ & $\mathrm{Zn}$ \\
\hline 4 & $\begin{array}{l}\text { Central Tennesee } \\
\text { district (E Imwood } \\
\text { and Gordonsville } \\
\text { mines; deposits } \\
\text { near Sugar Creek, } \\
\text { Gainesboro, } \\
\text { Hartsville, } \\
\text { Lebanon, } \\
\text { Carthage; six } \\
\text { sma11 mines on } \\
\text { veins, numerous } \\
\text { prospects and } \\
\text { occurrences) }\end{array}$ & $(\mathrm{Ba}, \mathrm{Zn}, \mathrm{Pb}$ \\
\hline
\end{tabular}

5 W. E. Bertram
No. 1, Sam Jarvis W. M. Phillips, No. 1, Bystoree, N.C.

\section{$\underline{\text { Size }}$}

Occurrence Lower Ordovician Knox Group

do.

do.

do.

Large

Lower Ordovician dolomite and limestone (Knox Group; most ore is in the

lower and middle

Mascot Dolomite;

subordinant amounts of ore are in the underlying Kingsport

Formation). Fissure

vein deposits are

mainly in Middle

Ordovician carbonate rocks, but are known in rocks as young as

the Lower Mississippian Warsaw Limestone.

Lower Ordovician Knox Group

do.

Lower Ordovician Knox Group

\section{Comments}

In we11 cuttings.

do.

do.

Predominant structures of large ore bodies are solution collapse breccias. Large ore deposits appear to be spatially associated with pre-Middle

Ordovician structure.

Fissure-vein deposits conta in barite, fluorite, and calcite with small amounts of sphalerite and galena.

In we11 cuttings.

do.

Do.
Selected references

Tennessee Division of Geology, 1969 , unpublished data.

Do.

Do.

Braun, E. R., 1983, p. 359; Callahan, 1977; Jewe 11, 1947: Kyle, 1976; Main. 1976; White, 1979; Winslow and Hill. 1973.

Tennessee Division of Geology, 1969, unpublished data. 
Map

number

Name

7

Pall Mall

Commodities

8

L. F. Choate

Smith

9 Grissom No. 1,

Pike $0 i 1$ Co.

10

Clemner-Roberson

No. 1, Sun 011

Company

11 Sewanee Fue 1 and Iron Company

No. 1, Sequatchie

Gas Company

Powell River

district (most

production from

the New Prospect,

Bunch Hollow and

Kings Bend mines.

Ninty-two mines.

prospects, and

occurrences

described)

13 Straight Creek district

(Straight Creek

mines, five

prospects)
$\mathrm{Ba}$

Size

Medium

\section{Host rock}

Residual clay overlying Mississippian limestone (St. Lou is Formation).

Zn

$\mathrm{Zn}$

Zn

$\mathrm{Zn}$

Sma 11

Cambrian 1 imestone and dolomite; the most productive deposits are in the Maynardville Limestone Member of the

Nolichucky Shale;

smaller deposits in

the Copper Ridge

Dolomite

$\mathrm{Zn}, \mathrm{Pb}$

do.

Cambrian dolomite

(Maynardville

Limestone and Copper

Ridge Dolomite)

\section{Comments}

Barite is restricted to a single favorable bed; no mineralization is known in the underlying limestones; possibly a bedded replacement deposit.

In we11 cuttings.

do.

do.

do.

Three types of deposits: (1) replacement deposits along faults, with some open-space filling in the breccia, (2) fracture fillings, and ( 3 ) bedded replacement deposits apparently unrelated to faults or fractures.

Ore is in a zone of crushed and broken dolomite in all gradations from small veinlets to large masses almost entirely replacing the country rock.
Maher, 1970 , p. 14-15.

Tennessee Division of Geology, 1969, unpublished data.

Do.

Do.

Do.

Brokaw, and others, 1966b.

Maher, 1958 , p. 2, 27; Secrist, 1924, p. 47-52. 
Map

number

Name

14

Evanston

district

(Livesay or

Mill cut,

six other

prospects)

Copper Ridge district (Ido and Flat Gap mines, eight prospects)

Valley mine

and prospect)

Click Ridge

Orebank mines and prospect

Barite nodule locality 6

\section{Commodities}

Zn

$\mathrm{Zn}$

Large

Lower Ordovician

dolomite and

limestone. (Mainly

in the Kingsport and

Mascot Formations)

Ba overlying lower Ordovician Mascot Dolomite

Prospect Cambrian limestone (Honaker Dolomite)

Sma 11

In residual clay

\section{Sma 11 \\ Barite in residual soils} overlying Lower

Ordovician dolomite (Upper Knox Group)

Occurrence

Middle Ordovician carbonaceous shale (Athens Shale)

\section{Comments}

Sma 11 replacement veinlets and disseminated flakes

and masses.

Principal ore zone is a brecciated dolomite. Brecciated zones are at facies boundaries between sediments that formed on topographic highs and those deposited in subtidal environments. Occurs in gangue of white, coarsely crystalline dolomite.

Brecciated horizons coincide with local strike of bedding.

Selected

references

Secrist, 1924, p. 53-64.

Churnet and

Misra, 1983;

Hi11, 1969;

Hill and others

1971; Hoagland

1962; Sayrs and

Clayton, 1949;

Secrist, 1924,

p. 80-96.

Maher, 1970, p. 30 .

Clear green crystalline barite in calcite vein.

Brobst and Hobbs, 1968, p. 274.

A small barite mine and a brown iron ore mine with abundant

Maher, 1970, p. 29. barite.

Nodules of barite and pyrite in black shale.
Carpenter and Fagan, 1969. 
Fall Branch-

$\mathrm{Ba}, \mathrm{Zn}$

Sma 11

district (ten mines that produced barite, about twenty-six barite prospects, Fall Branch Zinc Mine and eight zinc prospects)

Watauga Point and Carden

Nidifer Branch

(Barite prospect

in Stony Creek

District)

Dugger, Doughtery and Wagner zinc prospects; Draught Creek barite prospect and Dry Run Mine $(\mathrm{Mn}, \mathrm{Ba})$
Prospects

\section{Middle Cambrian Honaker Dolomite}

\section{$\mathrm{Ba}$}

$\mathrm{Mn}, \mathrm{Zn}, \mathrm{Ba}$ Prospe production from Dry Run Mine

$$
\begin{aligned}
& \mathrm{Fe}, \mathrm{Zn}, \\
& \mathrm{Pb}, \mathrm{Mn}
\end{aligned}
$$

Cambrian Shady

Dolomite and

its residuum

Cambrian Shady

Dolomite and its

residuum
Barite in residuum overlying carbonate rocks (mainly upper

Knox Group, but minor

mineralization in

carbonate rocks of the

Conasauga Group of

Cambrian age, and in

shales of Middle

Ordovician age).

Zinc in Lower

Ordovician dolomite

(Mascot and

Chepultepec Formations)

Barite in veins and crystalline aggregates in bedrock. Zinc in brecciated dolomite cemented by white, crystalline dolomite.

Replacement of country rock and fracturefillings.

Vein and fracture filling: residual barite and manganese deposits. In Butler and Stony Creek manganese districts.

Most production from oxidized deposits in residual clay; also

disseminated and associated with brecciated country rock and sparry

dolomite.
Dolomite and residual clay
Brokaw and others, 1966a, p. A17-A20; Maher, 1970 , p. 15-17 Secrist, 1924, p. 96-104.

Maher, 1958, p. 9-25; Secrist, 1924, p. 140-141.

Maher, 1970, p. 29; King and others, 1944 , p. 187.

King and others, 1944, p. 168-174: King and Ferguson, 1960, p. 86: Maher, 1970 , p. 17, 29.

Rodgers, 1948. 
Map

number

25

Name

Lost Creek

(Rhodelia)

Commodities Size

$\mathrm{Ba}(\mathrm{Zn}) \quad$ Small

26

$-$

$\mathrm{Ba}$ lower Kingsport
Host rock

In residual soil and underlying Middle Ordovician argillaceous dolomite (Dot

Formation) and Lower Ordovician dolomite (Mascot formation)

Prospect

In residuum of Lower Ordovician Upper Knox Group

Cambrian dolomite (Rome Formation)

In residuum above Lower Ordovician Knox Group (uppermost Longview Dolomite or

Formation

\section{Comments}

In stringers and blebs

\section{Lower Ordovician} leading zinc- dolomite (Mascot, producing Kingsport and upper district of Longview Formations the United of the Knox Group) States)
In a collapse breccia. Barite occurs as veins. coatings around breccia blocks, and fragments of coatings and veins embedded in argillaceous dolomite matrix. of white, crystalline calcite and in dolomite; dolomite has a shattered texture.

White barite in cherty residual clay.

McCormick and others, 1971: Harris, 1969 , p. 35-37; Fagan, 1969; Carpenter and others, 1971 . p. 792-795.

Maher, 1970, p. 32.

Maher and Finlayson, 1965 , p. 46 .

Maher and Finlayson, 1965 , p. 45.

Maher, 1958 p. 28.

Associated with white crystalline dolomite in solution-collapse breccias below the middle

Ordovician unconformity.
Fulweiler and McDougal, 1971; Harris, 1971; Hoag land, 1967: Hoagland and others, 1965; Kenda 1 1, 1960; Oder and Ricketts, 1961. Coy, North Friends Station, Jarnigan, Athletic, Beaver Creek mines) 
Barite nodule locality 1

Middle Ordovician carbonaceous shale (Athens Shale)

32

Barite nodule

$\mathrm{Ba}$

locality 2

33

Barite nodule

localities 3

and 4

34

Barite nodule locality 5

Naft prospect (Rader prospect, R. N. Hartman mine)

Kodak

Ba

do.

$37 \quad$ Kimberlin

do.

do.

do.

Ba (Zn) Sma11

Lower Ordovician dolomite (Mascot Formation)

do.

do.

do.

In residual clay above upper Knox Group (Kingsport Formation?)

38
$\mathrm{Pb}$

$\mathrm{Zn}(\mathrm{Fe})$

Trotter

Cambrian dolomite

(Rome Formation)

Prospect
Lower Ordovician slightly phosphatic carbonaceous dolomite (Mascot Formation) do.

Two ore-bearing horizons; one mostly of pyrite and barite forming narrow

seams and veinlets; one

with sphalerite in addition

to barite and pyrite, which

in places formed pockets.

\section{references}

Carpenter and Fagan, 1969.

Do.

Do.

Do.

Secrist, 1924, p. 102-104.

Maher, 1970, p. 32 .

Ramsey, 1926.

Disseminated in breccia matrix and fillings in solution cavities and channels of a paleokarst terrane, filling of bedding-plane fractures.
Carpenter and others, 1971. p. 796-797. 
Map

number

Name

Del Rio

District

(Eleven mines

and six prospects)

40 Blowing Cave

Ba

Ba, $\mathrm{Zn}$

41 Nuns Cove

$\mathrm{Ba}$

$$
\mathrm{Ba}, \mathrm{Zn}
$$

Commodities

In mylonite and breccia in fault zones: Cambrian Unicoi Formation) Precambrian quartzite (Snowbird Group); some in Precambrian slate and Lower Cambrian Erwin Formation

Residual clays overlying Lower (Knox Group)

Prospect

Residuum and dolomite of the Upper

Knox Group (Newala

Limestone)

42

Little
Mountain
(Fairgarden)

43

Chestnut Ridge

(W. Harrison)

44

do. Residuum and dolomite of the Upper Knox Group clay derived from Lower Ordovician Kingsport Formation

Lower Ordovician
C. C. Patrick quartzite (mostly Ordovician dolomite

Veins localized by overthrust faults and related structures.

son and Jewe 11, 1951; Maher, 1970, p. 13-14, 31 .

Possibly the first barite deposit noted in east Tennessee.

Kain, 1818; Maher, 1970, p. 32 .

Brobst and Hobbs, 1960, p. 274-275;

Maher and

Finlayson, 1965; p. 44-45; Maher, 1970, p. 17-18, 32.

Discrete blebs or crystalline barite and less abundant fine-grained sphalerite.

Maher, 1970 p. 17, 18, 32; Maher and Finlayson, 1965, p. 44-45.

White, medium- to coarsecrystalline barite masses with chert and clay.

Maher and Finlayson, 1965, dolomite (Knox Group) p. 45.

Maher, 1970 , p. 32; Secrist, 1924, p. 134.
Replacement and open-space filling of massively bedded, finely crystalline cherty dolomite. 
Map

number

45

Name

McReyno Ids

46

$\begin{aligned} & \text { Friendsville } \\ & \text { (Griffiths) }\end{aligned} \quad \mathrm{Ba}, \mathrm{Pb}, \mathrm{Fe}(\mathrm{F})$

47

J. F. Chapman

$\mathrm{Ba}(\mathrm{Fe}, \mathrm{Pb})$

भु do.

\section{Commodities}

$\mathrm{Ba}$

Prospect

Lower Ordovician

dolomite (Upper

Knox Group)

Residual clay

overlying Lower

Ordovician dolomite

(Mascot Formation) do.

do. do.

do.

\section{Comments}

Large and sma 11 pieces of barite on the surface.

Solution-collapse breccia. Barite is distributed throughout the mineralized horizon; galena and sphalerite occur in detached pockets in the brecciated zone, as

replacements of breccia fragments or small veinlets: oxidized ores occur in clay residuum.

Bedrock not exposed at surface.

Opened for lead about 1905. Dump contains barite, galena, and sphalerite. Secrist., 1924 p. $128-130$.

Maher, 1970, p. 32 .

p. 32 . 
Map

number

Name

Commodities

$\mathrm{Ba}(\mathrm{F}, \mathrm{Pb}, \mathrm{Zn})$

Sweetwater

District

(112 mines

and prospects

in 1960)

51

Notchy Creek

$\mathrm{Ba}$

W. L. Baugh

$\mathrm{Ba}$

53

Hambright

$\mathrm{Pb}$

$(\mathrm{Zn}, \mathrm{Ba}, \mathrm{Fe})$

Ba

(Cleveland)

55

Shofner

$\mathrm{Ba}$

Prospect
In residuum above

Lower Ordovician

Knox Dolomite

\section{Comments}

Nodules and larger masses of barite, chert, and limonite in reddish or yellow clay. Bedrock contains veins and breccia masses cemented by barite,

fluorite and pyrite with

some local concentrations of sphalerite and galena. Some structures that

localized barite thought to be produced by solution and some by tectonic movement (Maher, 1970, p. 13).

In residuum of Lower Ordovician Kingsport Formation

do. In residuum of very cherty Lower Ordovician Kingsport Formation

Lower Ordovician limestone and dolomite (Mascot Formation)

In residual clay overlying Lower Ordovician dolomite (principally Kingsport Formation and probably Mascot Formation)

Replacement veinlets in 1 imestone, no brecciation, minor amounts of coarsely crystalline dolomite.

Dump material consists chiefly of clay, chert, and barite and contains fragments of brecciated dolomite cemented by barite, chert and gangue dolomite.

Obscured by Highway 75 construction.
Selected

\section{references}

Laurence, 1960; Maher, 1970, p. 11-13.

Maher, 1970 , p. 34 .

Do.

Swing le, 1959 , p. 70-73.

Maher, 1970

p. 18-19;

Swingle, 1959 ,

p. 59-62.

Maher, 1970, p. 34 . 
Map

number

56

Name

Hardwick

(Dollie D)

Commodities

Size

Sma 11

$\mathrm{Pb}, \mathrm{Zn}$

(Ba)

Host rock

Upper Cambrian dolomite

(Maynardville

Limestone)
Selected

references

Dunlap, 1946; Swingle, 1959,

p. 73-75. shaped like an inverted funnel.

Open-space breccia filling
and replacement; deposit 


\section{Map}

number

1

Name

Frank 1 in

County

Commodities

$\mathrm{Zn}, \mathrm{Pb}$

\section{$\underline{\text { Size }}$}

Prospect

2 Lion Hill

(Oram, Brandon, Leicester)

赑

3 Orwell

4 Wallingford

5 Danby
$\mathrm{Pb}$

$\mathrm{Zn}, \mathrm{Pb},(\mathrm{Cu})$

do.

Upper Cambrian dolomite (Monkton or Winooski Formation)

\section{$\mathrm{Zn}$}

$\mathrm{Pb}$

\section{Host rock}

Lower Cambrian dolomite (Dunham Formation)
Comments

In highly deformed sedimentary breccia as matrix filling and solution breccia in favorable beds. Deformed by Taconic and Acadian orogenies.

In highly deformed folded and sheared autochthonous rock, mineralization deformed by Taconic orogeny (Ordovician).
Selected

references

J. P. Broderick, ora 1 commun., 1982.

Grant, 1968, p. 29-30; Jacobs, 1943(?), p. 18; Jacobs, 1944(?) p. 39; J. P. Broderick, oral commun., 1982.

Morrill and Chaffee, 1964, p. 33.

Morrill and Chaffee, 1964, p. 47.

Morrill and Chaffee, 1964, p. 15. 


\begin{tabular}{|c|c|c|c|c|c|c|}
\hline $\begin{array}{l}\text { Map } \\
\text { number }\end{array}$ & Name & Commodities & $\underline{\text { Size }}$ & Host rock & Comments & $\begin{array}{c}\text { Selected } \\
\text { references } \\
\end{array}$ \\
\hline 1 & Runion & $\mathrm{Zn}, \mathrm{Pb}$ & Prospect & Devonian sandstone & In veinlets of ankerite. & $\begin{array}{l}\text { Luttre11, 1966, } \\
\text { p. 114. }\end{array}$ \\
\hline 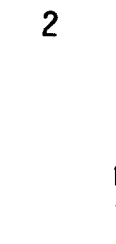 & $\begin{array}{l}\text { Shenandoah Valley } \\
\text { District } \\
\text { (Timberville and } \\
\text { Bowers-Campbell } \\
\text { mines and numerous } \\
\text { prospects) }\end{array}$ & $\mathrm{Zn}(\mathrm{Pb})$ & Medium & $\begin{array}{l}\text { Lower Ordovician } \\
\text { dolomite (upper } \\
\text { Beekmantown Group) }\end{array}$ & $\begin{array}{l}\text { With white, crystalline } \\
\text { dolomite in breccia-type } \\
\text { ore bodies, as vein } \\
\text { fillings and as isolated } \\
\text { replacement crystals. }\end{array}$ & $\begin{array}{l}\text { Brent, } 1960, \text { p. } 126- \\
132 \text {; Hayes, 1960; } \\
\text { Herbert and Young, } \\
\text { 1956; Young, 1956, } \\
\text { 1967. }\end{array}$ \\
\hline 3 & Grove $\mathrm{Hill}$ & $\mathrm{Zn}$ & Occurrence & $\begin{array}{l}\text { Lower Ordovician } \\
\text { dolomite (upper } \\
\text { Beekmantown Group) }\end{array}$ & $\begin{array}{l}\text { Associated with a minor } \\
\text { bedding plane fault; } \\
\text { disseminated crystals } \\
\text { replace thin gouge; larger } \\
\text { crystals are in vein } \\
\text { dolomite cementing } \\
\text { fractured beds. }\end{array}$ & $\begin{array}{l}\text { Herbert and Young, } \\
1956, \mathrm{p} .39 .\end{array}$ \\
\hline 4 & Wiseland & $\mathrm{Zn}$ & do. & $\begin{array}{l}\text { Lower Ordovician } \\
\text { dolomite (upper } \\
\text { Beekmantown Group) }\end{array}$ & $\begin{array}{l}\text { Small crystals in } \\
\text { "recrystalline" dolomite. }\end{array}$ & $\begin{array}{l}\text { Herbert and Young, } \\
1956, \text { p. } 36 .\end{array}$ \\
\hline 5 & Williamsville & $\mathrm{Ba}$ & Occurrence & $\begin{array}{l}\text { Devonian shale } \\
\text { (Millboro shale) }\end{array}$ & Barite nodules in shale. & $\begin{array}{l}\text { S. H. B. Clark, } \\
\text { this paper. }\end{array}$ \\
\hline 6 & Staunton & $\mathrm{Pb}, \mathrm{Zn}$ & Prospects & $\begin{array}{l}\text { Lower Ordovician } \\
\text { (Beekmantown Group) }\end{array}$ & $\begin{array}{l}\text { Sma11 prospects for } \\
\text { galena west of Staunton. }\end{array}$ & $\begin{array}{l}\text { Luttre11, } 1966, \\
\text { p. } 122 .\end{array}$ \\
\hline 7 & -- & $\mathrm{Ba}$ & -- & -- & -- & $\begin{array}{l}\text { Sweet and Rowe, } \\
1984, \text { p. } 8 \text {. }\end{array}$ \\
\hline 8 & $\begin{array}{l}\text { Earhart } \\
\text { prospect }\end{array}$ & $\mathrm{Ba}$ & Sma11 mine & -- & Residual barite in soil. & $\begin{array}{l}\text { Edmundson, } 1938, \\
\text { p. 80; Luttre 11, } \\
1966, \text { p. } 44 .\end{array}$ \\
\hline 9 & Clifton Forge & $\mathrm{Ba}$ & Occurrence & $\begin{array}{l}\text { Devonian shale } \\
\text { (Needmore Shale and } \\
\text { Millboro Shale } \\
\text { Members of the } \\
\text { Romney Shale) }\end{array}$ & $\begin{array}{l}\text { Barite concretions and } \\
\text { shrinkage cracks in } \\
\text { concretions filled with } \\
\text { veins of calcite, gypsum } \\
\text { and barite. }\end{array}$ & $\begin{array}{l}\text { Lesure, } F \text {. G. } \\
\text { 1957, p. 55-57. }\end{array}$ \\
\hline
\end{tabular}


Map

number

10

Becktel

Commodities

$\mathrm{Ba}$

$\mathrm{Ba}$

11

Shorter

$\mathrm{Ba}$

12

Johnson

13

Williamson

$\mathrm{Ba}$

14

Crush mine

and prospect

$\mathrm{Ba}$
17
Gus ler-Aust in and Reynold mines

$\mathrm{Ba}$

Huffman

Caldwe 11

Stony Creek

$\mathrm{Ba}$

Mason Cove
Size

do.

Prospect

Lower Ordovician

dolomi te

Beekmantown Group)

do.

In soil overlying

Lower Ordovician

dolomite (Beekmantown Group)

In soil derived from the Cambrian Conococheague Limestone

do.

Lower Ordovician

dolomite

(Beekmantown Group)

Prospect

Residual soil over-

lying Lower Ordovician

Beekmantown dolomite

Sma 11

Residual soil over-

dolomite (Beekman-

town Group) in the

Gusler-Austin Mine;

residual soil derived

from Cambrian

Conocheaque Limestone

in the Reynold mine

Prospect, Middle Ordovician

shallow

workings

Occurrence

$\mathrm{Ba}$
Athens Shale

Devonian shale

(Millboro Shale)

do.
Devonian shale (Romney Shale)

dolomite at the GuslerAustin Mine.

Fragments in residual

cement breccias in

In dolomite and in the overlying residual clay.

Residual deposit.

Associated with chert float.

In dolomite.

Residual deposit.

Residual deposit.

Edmundson, 1938 ,

1966 , p. 39 .

Edmundson, 1938,

p. 58-59;

Luttre11, 1966, p. 39.

Edmundson, 1938, p. 62; Luttre11, 1966 , p. 68.

Edmundson, 1938 , p. 60-62; Luttre11, 1966, p. $60,111$.
Dark-gray barite containing Edmundson, 1938 , carbonaceous matter occurs p. 60; Luttre11, in shale along a fault.

Float

1966 , p. 30 oral commun., 1983.

Nodules and in calcareous S. H. B. Clark, concretions in shale. this paper. 
Map
number

Name

Rucker mine

Commodities

$\mathrm{Ba}$

$\underline{\text { Size }}$

Sma 11

Host rock

In residual soil

overlying Lower

Ordovician

Beekmantown Group

21

Troutville

$\mathrm{Pb}, \mathrm{Zn}$

Prospect

Cambrian dolomite

(Elbrook Formation)

22

Reed mine

Ba

Sma 11

23

Bonsacks

$\mathrm{Zn}, \mathrm{Cu}$

Prospect

Martin and $\mathrm{Zn}, \mathrm{Pb}$
Wertz prospect

(Martin prospect)

Peppers Ferry

Mine

$\mathrm{Pb}, \mathrm{Zn}$

$\mathrm{Zn}$

Sma11

Prospect

26

Cloyd

$\mathrm{Zn}, \mathrm{Pb}$ Vaughn
(Rome Formation)

Brecciated

dolostone

Cambrian Rome or

Elbrook Formation?

Cambrian dolostone or limestone (Elbrook Formation) Cambrian dolostone
(Rome Formation)

Probably in dolomite of the Rome Formation (Cambrian)

do.

Cambrian dolostone

(Rome Formation)
Se lected

Comments

references

Edmundson, 1938, p. 62-63;

Luttre11, 1966, p. 113.

In dolomite breccia

cemented by white

dolomite.

As cement in breccia and replacement bodies along fractures and bedding planes.

Currier, 1935, p. 94; Luttre11, 1966, p. 132.

Luttre11, 1966, p. 110-111; Edmundson, 1938, p. 63.

Disseminated cavity fillings in brecciated dolomite veined with white dolomite.

In dolostone breccia cemented with white dolomite.

One solid mass of lead ore and small pockets.

Zinc ore reported.

Luttre11, 1966, p. 22; Watson, 1905 , p. 73, 1907 , p. 530 Woodward, 1932, p. 116 .

Luttre11, 1966, p. 91; Watson, 1905 , p. 71-72, 1907 , p. 530; Woodward, 1932 , p. 116.

Luttre11, 1966, p. 104.

Currier, 1935, p. 95; Luttre11, 1966 , p. 35; Watson, 1905, p. 75-76.

Currier, 1935, p. 95; Luttre11, 1966 , p. 34.

Currier, 1935, p. 95; Luttre 11 1966, p. 138. 
(Bonys Run)

30 Camp Kiwanianna

$\mathrm{Zn}, \mathrm{Cu}$

Prospect (Piedmont)
Cambrian dolostone (Rome Formation?)

Cambrian dolostone (Shady Dolomite)

do.

Disseminated in white dolomite and cementing fractures in gray dolomite.

Disseminated.

Disseminated splotches and veins in breccia and replacement of carbonate cementing the basal sandstone of the Shady Dolomite.

\section{Disseminated.}

Disseminated and filling fractures in breccia.

Zinc minerals reported from 2 mi les east of Springville near the end of Taylors Ridge.

do.

Limestone, probably of Devonian age

do. Cambrian limestone (Shady Dolomite)

Cambrian 1 imestone (Nolichucky Shale)
Associated with pyrite and oxides of iron and manganese.

Sunbursts of barite occassionally, accessory pyrite.

South of a major overthrust fault.
Currier, 1935 , p. 95; Dietrich, 1954, p. 28; Luttre11, 1966, p. 79; Watson, 1905, p. 75-76; Woodward, 1932, p. 116.

Dietrich, 1954, p. 30; Luttre11, 1966, p. 31 .

Dietrich, 1954, p. 28-30, 1959, p. 150; Luttre11, 1966, p. 129-130.

Luttre11, 1966, p. 7 .

Fontaine, 1883, p. 190; Luttre11, 1966, p. 105-106.

Currier, 1935 , p. 109; Luttre11, 1966, p. 127.

Currier, 1935, p. 109; Luttre11, 1966, p. 78.

Windolph, 1985a, 1985b.

Currier, 1935 , p. 109. 
Lower Ordovician

limestone and

dolomite

(Beekmantown Group)

39

Orr

40

Smyth County area

Ba (Cu) Smal1

$\mathrm{Ba}$

Medium

$\mathrm{Pb}, \mathrm{Zn}$

(Mn, Pyrite, $A u)$

Smal1

Lower Ordovician

dolomite

(Beekmantown Group)

Lower Ordovician

limestone and

dolomite

(Beekmantown Group)

Cambrian dolostone

(Shady Formation)
Rye Valley
District (Rye
Valley Mining
Company Mine;
R. N. Ward,
Livesay,
Calhon, Martin,
Scott, Van Hoy
Prospects)

Cedar Springs

District (Cedar

Springs mine;

James, Davis,

Bennington,

Wilkinson

properties,

Porter Branch

mine)

43

Mount Ephraim

Church (Sand

Mountain area)

44 J. C.

Groseclose
$\begin{array}{lll}\mathrm{Zn} & \text { Smal1 } & \begin{array}{l}\text { Cambrian dolostone } \\ \text { (Shady Formation) }\end{array}\end{array}$

Zn Prospect Cambrian dolostone

(Shady Formation)

Ba Prospect
Fracture fillings and

replacement masses in

bedrock and irregular

fragments in the overlying

residual soil.

Replacement masses.

Veins and replacement masses in bedrock and irregular fragments in

the overlying residual soil.

In brecciated zones cemented by coarsegrained white dolomite.

In dolomite veins in crackle and shatter

breccia, also in residual iron deposit. p. 100-105, 1907,

In breccia with veins of coarse white dolomite and calcite.

Abundant fragments of barite float.
Currier, p. 105106; Watson, 1905 p. 538-540.

Brobst, 1965, p. 8; Edmundson, 1938 . p. 63-76.

Edmundson, 1938 , p. 79; Luttre11, 1966, p. 101.

Brobst, 1965 , p. 8; Edmundson, 1938, p. 76-79.

Currier, 1935, p. 106-109; Watson, 1905 , p. 105-113, 1907, p. 540-541.

Currier, 1935, p. 104.

Edmundson, 1938, p. 80; Luttre11, 1966, p. 59. 


\begin{tabular}{|c|c|c|c|c|c|c|}
\hline $\begin{array}{l}\text { Map } \\
\text { number }\end{array}$ & Name & Commodities & $\underline{\text { Size }}$ & Host rock & Comments & $\begin{array}{c}\text { Selected } \\
\text { references }\end{array}$ \\
\hline 45 & Taylor & $\mathrm{Zn}(\mathrm{Pb})$ & Prospect & $\begin{array}{l}\text { Cambrian dolostone } \\
\text { (Shady Formation) }\end{array}$ & $\begin{array}{l}\text { In breccia carrying } \\
\text { white dolomite and quartz. }\end{array}$ & $\begin{array}{l}\text { Currier, } 1935, \\
\text { p. } 105 .\end{array}$ \\
\hline 46 & $\begin{array}{l}\text { Cripple Creek } \\
\text { area }\end{array}$ & (Fe, Pyrite) & Sma 11 & $\begin{array}{l}\text { Cambrian dolostone } \\
\text { (Shady Formation) }\end{array}$ & $\begin{array}{l}\text { In residual iron deposits; } \\
\text { also reported in dolomite. }\end{array}$ & $\begin{array}{l}\text { Currier, } 1935, \\
\text { p. 103-104. }\end{array}$ \\
\hline 47 & $\begin{array}{l}\text { Austinville- } \\
\text { Ivanhoe } \\
\text { District } \\
\text { (Austinville } \\
\text { and Ivanhoe } \\
\text { mines, } \\
\text { numerous small } \\
\text { mines and } \\
\text { prospects) }\end{array}$ & $\mathrm{Zn}, \mathrm{Pb}$ & Large & $\begin{array}{l}\text { Lower Cambrian } \\
\text { dolomi te } \\
\text { (Shady Dolomite) }\end{array}$ & $\begin{array}{l}\text { In breccias and as } \\
\text { replacement of sedimentary } \\
\text { features; localization } \\
\text { of ore is thought to be } \\
\text { at facies boundary zones. }\end{array}$ & $\begin{array}{l}\text { Brown, 1935, 1953; } \\
\text { Brown and Weinburg, } \\
\text { 1968; Currier, 1935, } \\
\text { p.99-103; Luttre11, } \\
1966, \text { p. 12-13, 71; } \\
\text { Stose and Stose, } \\
1957, \text { p. 210; } \\
\text { Watson, 1905, p. 83- } \\
99,1907, \text { p. 532-538. }\end{array}$ \\
\hline 48 & $\begin{array}{l}\text { Bertha District } \\
\text { (Bertha, Barren } \\
\text { Springs and } \\
\text { Falling Cliff } \\
\text { mines, several } \\
\text { other pits) }\end{array}$ & $(\mathrm{Fe}, \mathrm{Zn}$ & Sma 11 & $\begin{array}{l}\text { Residual clay } \\
\text { overlying the } \\
\text { Cambrian Shady } \\
\text { Dolomite }\end{array}$ & $\begin{array}{l}\text { Residual clays worked; } \\
\text { disseminated sphalerite } \\
\text { only in small amounts } \\
\text { in underlying rocks, which } \\
\text { lack brecciation. }\end{array}$ & $\begin{array}{l}\text { Currier, 1935, p. 97- } \\
\text { 98; Luttrel1, 1966, } \\
\text { p. 18-19; Watson, } \\
1905, \text { p. 78-83, } \\
\text { 1907, p. 532. }\end{array}$ \\
\hline 49 & $\begin{array}{l}\text { Allisonia } \\
\text { District (Delton } \\
\text { mines, Forney, } \\
\text { Graham and } \\
\text { Robinson and } \\
\text { Flanigan } \\
\text { prospects) }\end{array}$ & $\mathrm{Zn}, \mathrm{Pb}, \mathrm{Fe}$ & Sma 11 & $\begin{array}{l}\text { Cambrian dolostone } \\
\text { (Rome and Shady } \\
\text { Formation }\end{array}$ & $\begin{array}{l}\text { Zinc mined from oxidized } \\
\text { zone; also in breccia } \\
\text { with white dolomite } \\
\text { cement. }\end{array}$ & $\begin{array}{l}\text { Currier, } 1935, \\
\text { p. 95-97; Watson, } \\
1905, \text { p. } 75, \\
1907 \text {, p. 531. }\end{array}$ \\
\hline 50 & Brickey (Hagan) & $\mathrm{Zn}$ & Prospect & $\begin{array}{l}\text { Cambrian dolostone } \\
\text { (Rome Formation) }\end{array}$ & Vein/shear zone. & $\begin{array}{l}\text { Sweet and Rowe, } \\
1984, \text { p. } 7,26 .\end{array}$ \\
\hline 51 & Pat Keith & $\mathrm{Zn}, \mathrm{Pb}$ & Prospect & $\begin{array}{l}\text { Cambrian dolostone } \\
\text { (Copper Ridge } \\
\text { Dolomite) }\end{array}$ & $\begin{array}{l}\text { Disseminated smal1 } \\
\text { masses. }\end{array}$ & $\begin{array}{l}\text { Luttre11, 1966, } \\
\text { p. 103-104; } \\
\text { Woodward, 1938, } \\
\text { p. } 69 .\end{array}$ \\
\hline
\end{tabular}


Sma 11

Middle Cambrian

limestone (Maryville Limestone)

Possibly near the trace of a major thrust fault.

Middle Ordovician Holston Limestone

Bowman (BowmanGraves, Arcadia, Graves)

Cambrian dolostone (Rome Formation)

\section{Sma 11}

Cambrian dolostone (Honaker Formation and Copper Ridge Dolomite)
$\mathrm{Ba}$
Seams contained smal1 quantities of calamine in thin, irregular zones along the bedding of the rocks.

Disseminated near a zone of thrust faulting.

In white dolomite

in a brecciated zone near a thrust fault.

Nodules of barite and pyrite in black shale.
Middle Ordovician carbonaceous shale (Athens Shale)
Currier, 1935 , p. 109; Luttrel1, 1966, p. 101; Watson, 1905, p. 113-116;

Woodward, 1938, p. 67-69.

Luttre11, 1966, p. 92.

Brent, 1963, p. 42; Luttre 11, 1966, p. 79 .

Currier, 1935 , p. 110; Luttre11, 1966, p. 24; Secrist, 1924 , p. 33-37.

\section{Carpenter and}

Fagan, 1969

p. 17-29. 


\section{Map \\ number}

1 Monongahela River

2 Tunnelton

3 Parsons

4 Knobly Mountain

5 Dry Run of Mill Creek

6 Romney

7 Howe 11 farm

\section{Commodities}

$\mathrm{Zn}$

$\mathrm{Ba}(\mathrm{Pb})$

$\mathrm{Zn}$

$\mathrm{Zn}$

$\mathrm{Pb}$

$\mathrm{Ba}$

$\mathrm{Zn}(\mathrm{Pb})$

\section{Size}

Occurrence

\section{Host rock}

Pennsylvanian Brush Creek Shale

do. Pennsylvanian

do.

A lens of coal
in Devonian
sandstone

do. Devonian Oriskany Sand stone

do.

Upper Devonian alternating flagstone and shale. Basal Chemung or upper

Portage Formation.

do. Devonian shale

Prospect
Devonian shale

Cambrian dolomitic limestone (Tomstown Formation)
Selected references

Martens, 1964,

Sphalerite is found in the cores of small siderite nodules.

Barite and a very sma 11 amount of galena in siderite concretions about 15 feet below the Upper Freeport Coal.

Thin veins of light brown sphalerite along joints in a lens of coal: much pyrite associated with the coal.

Light-brown sphalerite, mostly in small grains occurs in a block of

sandstone at the base of a cliff composed of Oriskany Sandstone.

In thin quartz veinlets.

Reger and Tucker 1924, p. 708.

Barite in veins in large limestone concretions in shale.

Martens, 1963, p. 4 ; 1964 , p. 31 .

Breccia filling along bedding.

Ludlum, 1955 , p. 855-861. 
number Name

8 Hopeville

$9 \quad$ Petersburg

10 Moorefield

11 Riverton

12 Jericho Draft

\section{Commodities}

$\mathrm{Ba}$

Ba (?)

$\mathrm{Ba}$

$\mathrm{Ba}$

$\mathrm{Zn}$

\section{Size} Occurrence

\section{Host rock} Shale

do.

Devonian shale

do.

do.

Occurrence

\section{Devonian Ridgley}

Devonian Marcellus

\section{Comments}

Barite and pyrite in 1 imestone nodules in

shale.

In a large septarium concretion.

In concretions.

do.

Disseminated sphalerite(?)

in calcareous sandstone.
Selected

references

Martens, 1964, p. 29-30.

Johnson, 1961, p. 58; Martens, 1963 , p. 4.

Martens, 1963 p. 4 .

Do.

Sandstone
Lesure and others, 1982. 
Map

number

1

\section{Name}

Commodities

Upper Mississippi Valley lead-zinc

district (The

district includes

313 mines in

Wisconsin.

Subdistricts in

Wiscons in are

Haze1 Green-

Shullsburg,

Meekers Grove

(Jenk insville),

Big Patch,

Plattsvilie,

Cal amine-Truman-

Darlington,

Potosi, Fairplay,

Beetown, Fennimore,

Mifflin-Cokerville,

and Mineral Point-

Linden-Dodgeville).

This is the same

locality as site 1

of Illinois.

Little Kickapoo
lead diggings

$\mathrm{Pb}, \mathrm{Cu}$

Sma 11

Large

Host rock

Midd le Ordovician

limestone and dolomite

(most deposits in

Galena Dolomite,

Decorah Formation, and Platteville

Formation); deposits of sulfides found in al1 formations exposed within the mineralized part of the district.

Lower Ordovician

silicified and

brecciated dolomite

(Oneota Dolomite,

Prairie du Chien Group)
$\mathrm{Pb}, \mathrm{Cu}$ lead diggings
Lower Ordovician

Prairie du Chien

Group

\section{Comments}

Ore bodies can be classified as (1) reverse-fault and fold controlled ore bodies, (2) joint-controlled ore bodies, and (3)

placer and residual deposits. The ores in the fault and fold deposits occur as (1) vein fillings along fractures and bedding planes; (2) cavity fillings in solution breccias; and (3) disseminations by replacement and impregnation in favorable beds, particularly in shaly strata. Joint controlled deposits are veins in vertical joints and podlike deposits in beds crossed by joints (Hey 1 and others, 1959).

\section{As large crystals and} masses.

Similar to Little Kickapoo lead diggings (3).
Se lected

references

Hey 1 and others 1959; Hey 1, 1968a, Hey 1 and West. 1982, p. 1803-1807; McLimans, 1977.
Heyl and others 1959 , p. 294; Hey 1 and West, 1982, p. 1804, 1806; Strong, 1882, p. 75-78.

Heyl and others, 1959 , p. 294; Hey 1 and West, 1982 , p. 1804,1806 . 


\section{Map}

number

4

Name

Woodman lead

mine

Commodities

$\mathrm{Pb}, \mathrm{Cu}$

5

Bridgeport $\quad \mathrm{Pb}, \mathrm{Zn}$

quarry

6

Little Grant

lead diggings

$\mathrm{Pb}$

Sma 11

7

Castle Rock

$\mathrm{Pb}$

Occurrence

8

Keyesville lead diggings

9

Akan lead

mine

10

Orion lead mine

$\mathrm{Pb}, \mathrm{Cu}$

do.

do.

Sextonville lead diggings

$\mathrm{Pb}, \mathrm{Cu}$

$\mathrm{Pb}$

Occurrence
Lower Ordovician

Prairie du Chien Group; markedly silifified by jasperoid.

Lower Ordovician brecciated dolomite (Oneota Dolomite, Prairie du Chien Group)

Lower Ordovician Prairie du Chien Group
Host rock

Comments

Workings follow fractures

and brecciated areas along which galena and calcite were deposited.

As replacements, crystals 1 ining vugs, and veinlets in breccia.

In vertical fractures associated with abundant iron sulfides.

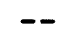

do.

do.

do.

do.

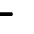

In openings in silicified rock.

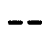

Do.

Do.

Hey 1 and others, 1959, p. 294; Heyl and West, 1982 , p. 1804 .

Hey 1 and others, 1959 , p. 293; Hey 1 and West, 1982 , p. 1804 .

Heyl and others, 1959, p. 295; Hey 1 and West, 1982 , p. 1804 .

Do.

Do.

Do.

Hey 1 and others, 1959, p. 293; Heyl and West, 1982, p. 1804, 1806 . 
Map

number

13

Name

Demby-Weist

mines

14

Black Earth

lead diggings

15

Pine Bluff

lead diggings

16 Lodi

17 Rio lead

diggings

18 Cambria

19 Doylestown

20 Columbus

21 Fitchburg

22 Avon

23 Clarno

\section{Commodities}

$\mathrm{Pb}, \mathrm{Zn}$

Size

Smal1

Host rock

Lower Ordovician

Prairie du Chien Group and Jordan Sandstone member of the Upper

Cambrian Trempea leau Formation.

Pb

do. Lower Ordovician

Prairie du Chien Group

do.

Occurrence

Sma 11

do.

do.

Occurrence

do.

do.

do.
Worked about 1828.

\section{Comments}

Veins and lodes in vertical or steeply dipping faults or fissures.

do.
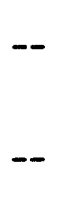

$-$

$--$

$-$

$-$

$-$

Hey 1 and others,

1959 , p. 280-282;

Hey 1 and West,

1982, p. 1804,

1806.

Hey 1 and others, 1959, p. 295; Hey 1 and West, 1982 ,

p. 1804,1806 .

Do.

Hey 1 and West, 1982 , p. 1804 .

Heyl and others. 1959, p. 295; Heyl and West, 1982 , p. 1804 .

Do.

Do.

Hey 1 and West, 1982 , p. 1804 .

Do.

Do.

Hey 1 and others, 1959, p. 293; Hey 1 and West, 1982 , p. 1804 . 


\section{REFERENCES CITED}

Adams, G. I., and Jones, W. B., 1940, Barite deposits of Alabama: Alabama Geological Survey Bulletin 45, 38 p.

Anderson, G. M., 1973, The hydrothermal transport and deposition of galena and sphalerite near $100^{\circ} \mathrm{C}$ : Economic Geology, v. 68, p. $480-492$.

1975. Precipitation of Mississippi Valley type ores: Economic Geology, v. 70, p. 937-942.

1983, Some geochemical aspects of sulfide precipitation in carbonate rocks, in Kisvarsanyi, Geza, Grant, S. K., Pratt, W. P., and Koenig, J. W., ed., International conference on Mississippi Valley type lead-zinc deposits: Rolla, Missouri, University of Missouri-Rolla, p. 61-76.

Anderson, G. M., and Macqueen, R. W., 1982, Ore deposits models-6. Mississippi Valley type lead-zinc deposits: Geoscience Canada, v. 9 , p. 108-117.

Barnes, H. L., 1983, Ore-depositing reactions in Mississippi Valley-type deposits, in Kisvarsanyi, Geza, Grant, S. K., Pratt, W. P., and Koenig, J. W., ed., International Conference on Mississippi Valley type lead-zinc deposits: Rolla, Missouri, University of Missouri-Rolla, p. 77-85.

Bassett, W. A., and Kinsland, G. L., 1973, Mineral collecting at Penfield Quarry, in Hewitt, P. C., ed., Guidebook to field trips: New York State Geological Association 45th Annual Meeting, p. H1-H9.

Bastin, E. S., and others, 1939, Contributions to a knowledge of the lead and zinc deposits of the Mississippi Valley region: Geological Society of America Special Paper 24, $156 \mathrm{p}$.

Bayles, R. E., 1936, Minerals of West Virginia: West Virginia University, M.S. thesis, $42 \mathrm{p}$.

Beales, F. W., 1975, Precipitation mechanisms for Mississippi Valley-type ore deposits: Economic Geology, v. 70, p. 943-948.

Beales, F. W., and Jackson, S. A., 1966, Precipitation of lead-zinc ores in carbonate reservoirs as illustrated by Pine Point ore field, Canada: Institution of Mining and Metallurgy Transactions, section B, v. 75, Bulletin 720, p. B278-B285.

Beck, L. C., 1842, Mineralogy of New York: Albany, N.Y., 534 p.

Berkheiser, S. W., Jr., 1984, Fetid barite occurrences, western Berks County, Pennsylvania: Pennsylvania Geological Survey Mineral Resources Report 84, 43 p.

Boctor, N. Z., Kullerud, Gunnar, and Sweany, J. L., 1976, Sulfide minerals in Seelyville coal bed III, Chinook Mine, Indiana: Mineralium Deposita, v. 11, no. 3, p. 249-266.

Botoman, George, and Stieglitz, R. D., 1978, The occurrence of sulfide and associated minerals in Ohio: Ohio Geological Survey Report of Investigations $104,11 \mathrm{p}$.

Bradbury, J. C., 1957, Outlying occurrences of galena, sphalerite, and fluorite in Illinois: Illinois Geological Survey Industrial Minerals Note $7,5 \mathrm{p}$.

1959, Crevice lead-zinc deposits of northwestern Illinois: Illinois State Geological Survey Report of Investigation 210, 49 p.

Braun, E. R., 1983, Ore controls-Middle Tennessee zinc district, in Kisvarsanyi, Geza, Grant, S. K., Pratt, W. P., and Koenig, J. W., ed., International conference on Mississippi Valley type lead-zinc deposits: Rolla, Missouri, University of Missouri-Rolla, p. 349-359.

Brent, W. B., 1960, Geology and mineral resources of Rockingham County: Virginia Division of Mineral Resources Bulletin 76, $174 \mathrm{p}$.

1963, Geology of the Clinchport quadrangle, Virginia: Virginia Division of Mineral Resources Report of Investigations 5, p. 42.

Brobst, D. A., 1965, Barite in the United States, exclusive of Alaska and Hawaii: U.S. Geological Survey Miscellaneous Investigations Resource Map MR-43, scale 1:31,680.

Brobst, D. A., and Hobbs, R. G., 1968, Barite, in U.S. Geological Survey and U.S. Bureau of Mines, Mineral Resources of the Appalachian Region: U.S. Geological Survey Professional Paper 580, p. 270 277.

Brokaw, A. L., Dunlap, J. C., and Rodgers, John, 1966a, Geology and mineral deposits of the Mosheim and Johnson anticlines, Greene County, Tennessee: U.S. Geological Survey Bulletin 1222-A, p. A1-A21.
Brokaw, A. L., Rodgers, John, Kent, D. F., Laurence, R. A., and Behre, C. H., Jr., 1966 b, Geology and mineral deposits of the Powell River area, Claiborne and Union Counties, Tennessee: U.S. Geological Survey Bulletin 1222-C, p. C1-C56.

Brown, C. E., 1983, Mineralization, mining, and mineral resources in the Beaver Creek area of the Grenville lowlands in St. Lawrence County, New York: U.S. Geological Survey Professional Paper $1279,21 \mathrm{p}$.

Brown, J. S., ed., 1967, Genesis of stratiform lead-zinc-barite-fluorite deposits in carbonate rocks (the so-called Misssissippi Valley type deposits)-A symposium, New York, "1966: Economic Geology Monograph 3, $433 \mathrm{p}$.

1970, Mississippi Valley type lead-zinc ores; a review and sequel to the "Behre Symposium:" Mineralium Deposita: v. 5, p. 103-119.

Brown, W. H., 1935, Quantitative study of ore zoning, Austinville mine, Wythe County, Virginia: Economic Geology, v. 30, no. 4, p. 425433.

1953, The geology [Austinville basin, Virginia]: Mining Engineering, v. 5, no. 12, p. 1216-1221.

Brown, W. H., and Weinberg, E. L., 1968, Geology of the AustinvilleIvanhoe District, Virginia, in Ridge, J. D., ed. Ore deposits of the United States, 1933-1967, v. 1: New York, American Institute of Mining, Metallurgical, and Petroleum Engineers, p. 169-186.

Buddington, A. F., 1934, Geology and mineral resources of the Hammond, Antwerp and Lowville Quadrangles: New York State Museum Bulletin 296, p. 202-209.

Butts, Charles, and Gildersleeve, Benjamin, 1948, Geology and mineral resources of the Paleozoic area in northwest Georgia: Georgia Geological Survey Bulletin 54, 176 p.

Callahan, W. H., 1968, Geology of the Friedensville zinc mine, Lehigh County, Pennsylvania, in Ridge, J. D., ed., Ore deposits of the United States; 1933-1967, v. 1: New York, American Institute of Mining, Metallurgical, and Petroleum Engineers, v. 1, p. 95-107.

Callahan, W. H., 1977, The history of the discovery of the zinc deposit at Elmwood, Tennessee-concept and consequence: Economic Geology, v. 72, p. 1382-1392.

Cannon, H. L., 1955, Geochemical relations of zinc-bearing peat to the Lockport Dolomite, Oreleans County, New York: U.S. Geological Survey Bulletin 1000-D, p. 119-185.

Carlson, E. H., 1983, The occurrence of Mississippi Valley-type mineralization in northwestern Ohio, in Kisvarsanyi, Geza, Grant, S. K., Pratt, W. P., and Koenig, J. W., ed., International conference on Mississippi Valley type lead-zinc deposits: Rolla, Missouri, University of Missouri-Rolla, p. 424435.

Carpenter, R. H., and Fagan, J. M., 1969, Barite nodules in the Athens Shale in northeastern Tennessee and southwest Virginia: Southeastern Geology, 1969, v. 10, p. 17-29.

Carpenter, R. H., Fagan, J. M., and Wedow, Helmuth, Jr., 1971, Evidence on the age of barite, zinc, and iron mineralization in the Lower Paleozoic rocks of east Tennessee: Economic Geology, v. 66. no. 5 , p. $792-798$.

Cathles, L. M., and Smith, A. T., 1983, Thermal constraints on the formation of Mississippi Valley-type lead-zinc deposits and their implications for episodic basin dewatering and deposit genesis: Economic Geology, v. 78, p. 983-1002.

Churnet, H. G., and Misra, K. C., 1983, Sphalerite mineralization and its relationship to carbonate facies boundaries, Cooper Ridge district, east Tennessee, in Kisvarsanyi, Geza, Grant, S. K., Pratt, W. P., and Koenig, J. W., ed., International Conference on Mississippi Valley type lead-zinc deposits: Rolla, Missouri, University of Missouri-Rolla, p. $360-372$.

Clark, S. H. B., and Neeley, C. L., 1983, Zinc, lead, and barite deposits and occurrences in Paleozoic rocks, east central United States: U.S. Geological Survey Open-File Report 83-187, 79 p. and 1 sheet.

Cobb, J. C., 1981, Geology and geochemistry of sphalerite in coal: Champlain, Illinois, Illinois State Geological Survey, $204 \mathrm{p}$

Cobb, J. C., Steele, J. D., Treworgy, C. G., and Ashby, J. F., 1980, The abundance of zinc and cadmium in sphalerite-bearing coals in Illinois: Illinois Geological Survey Industrial Minerals Note 74, 28 p. 
Cook, R. B., 1978, Minerals of Georgia-their properties and occurrences: Georgia Geological Survey Bulletin 92, $189 \mathrm{p}$.

Coveney, R. M., and Martin, S. P., 1983, Molybdenum and other heavy metals of the Mecca Quarry and Logan Quarry Shales: Economic Geology, v.78, p. 132-149

Crawford, M. J., and Beales, F. W., 1983, The Shawangunk mine: a possible sandstone-hosted Mississippi Valley-type ore deposit in New York, in Kisvarsanyi, Geza, Grant, S. K., Pratt, W. P., and Koenig, J. W., ed., International Conference on Mississippi Valley type leadzinc deposits: Rolla, Missouri, University of Missouri-Rolla, p. 436445 .

Currier, L. W., 1923, Fluorspar deposits of Kentucky: Kentucky Geological Survey, series 6, v. 13, 198 p.

1935, Zinc and lead region of southwestern Virginia: Virginia Geological Survey Bulletin 43, 122 p.

Currier, L. W., 1937, Origin of the bedding replacement deposits of fluorspar in the Illinois field: Economic Geology, v. 32, p. 364-386.

Dale, N. C., 1953, Geology and mineral resources of the Oriskany quadrangle: New York State Museum Bulletin 345, p. 103-108.

Davis, C. A., 1909, Report on the geology of Tuscola County, Michigan: Michigan Geological Survey Annual Report 1908, pt. C, p. 125353

Desborough, G. A., 1959, Sulphide mineralization and associated structure in northern Union County, Illinois: Washington Academy of Sciences Journal, v. 49, no. 6, p. 172-177.

DeWitt, Wallace, Jr., 1974, Geologic map of the Beans Cove and Hyndman quadrangles and part of the Fairhope quadrangle, Bedford County, Pennsylvania: U.S. Geological Survey Miscellaneous Investigations Series Map I-801, scale 1:24,000.

Dietrich, R. V., 1954, Geology of the Pilot Mountain area, Virginia: Bulletin of the Virginia Polytechnic Institute, Engineering Station Series 91 , v. 47 , no. 4,32 p.

1959, Geology and mineral resources of Floyd County of the Blue Ridge Upland, southwestern Virginia: Bulletin of the Virginia Polytechnic Institute, Engineering Experiment Station Series 134, v. 52 , no. $12,160 \mathrm{p}$.

Dorr, J. A, Jr., and Eschman, D. F., 1970, Geology of Michigan: Ann Arbor, Michigan, University of Michigan Press, $476 \mathrm{p}$.

Dozy, J. J., 1970, A geological model for the genesis of the lead-zinc ores of the Mississippi Valley, U.S.A.: Transactions of the Institute of Mining and Metallurgy, v. 79, p. B163-B170.

Dunlap, J. C., 1945, Barite prospects in the vicinity of Friendsville, Blount County, Tennessee: U.S. Geological Survey Strategic Minerals Investigations Preliminary Report 3-191.

1946, Geology of Hardwick mine area, Bradley County, Tennessee: U.S. Geological Survey Strategic Minerals Investigation Preliminary Report 3-185.

Edmundson, R. S., 1938, Barite deposits of Virginia: Virginia Geological Survey Bulletin 53, $85 \mathrm{p}$.

Eilertsen, N. A., 1950, Investigation of the Shawangunk mine zinc-lead deposit, near Summitville, Sullivan Co., N.Y.: U.S. Bureau of Mines Report of Investigations 4675, $41 \mathrm{p}$.

Erd, R. C., and Greenberg, S. S., 1960, Minerals of Indiana: Indiana Geological Survey Bulletin 18, 73 p.

Ettensohn, F. R., and Barron, L. S., 1981, Depositional model for the Devonian-Mississippian black shales of America: A paleoclimaticpaleogeographic approach, in Roberts, T. G., ed., Chattanooga and Ohio Shales of the southern Appalachian Basin: Geological Society of America Guidebook, v. 2, p. 344-367.

Fagan, J. M., 1969, Geology of the Lost Creek barite mine: Tennessee Department of Conservation, Division of Geology Report of Investigations 23, p. 40-44.

Ferguson, H. W., and Jewell, W. B., 1951, Geology and barite deposits of the Del Rio district, Cocke County, Tennessee: Tennessee Department of Conservation, Division of Geology Bulletin 57, $235 \mathrm{p}$.

Fohs, F. J., 1913, Barytes deposits of Kentucky: Kentucky Geological Survey, series 4 , v. 1 , p. $441-588$.
Fontaine, W. M., 1883, Notes on the geology and mineral resources of the Floyd, Virginia, plateau: The Virginias, v. 4, p. 190.

Freedman, J. L., 1972, Geochemical prospecting for zinc, lead, copper, and silver, Lancaster Valley, southeastern Pennsylvania: U.S. Geological Survey Bulletin 1314-C, 49 p.

Fulweiler, R. E., and McDougal, S. E., 1971, Bedded-ore structures, Jefferson City mine, Jefferson City, Tennessee: Economic Geology, v. 66, p. $763-769$.

Garven, Grant, and Freeze, R. A., 1982, The role of regional groundwater flow in the formation of ore deposits in sedimentary basins: a quantitative analysis, in Ozoray, G., ed., Proceedings of the Second National Hydrogeological Conference, sponsored by the International Association of Hydrogeologists, Canadian Chapter, p. $59-68$.

Giordano, T. H., and Barnes, H. L., 1981, Lead transport in Mississippi Valley-type ore solutions: Economic Geology, v. 76, p. 2200-2211.

Grant, R. W., 1968, Mineral collecting in Vermont: Vermont Geological Survey Special Publication 2, $49 \mathrm{p}$.

Gray, Carlyle, 1961, Zinc and lead deposits of the Shawangunk Mountains, New York: New York Academy of Science Transactions Series 2, v. 23, p. 315-331.

Green, Richard, 1971, Ohio Lime, Woodville: Rocks and Minerals, v. 46, p. 280-281.

Greene, G. U., 1935, The occurrence of sphalerite at Ellsworth, Ohio: American Mineralogist, v. 20, p. 882-883.

Grogan, R. M., and Bradbury, J. C., 1968, Fluorite-zinc-lead deposits of the Illinois-Kentucky mining district, in Ridge, J. D., ed., Ore deposits of the United States, 1933-1967, v. 1: New York, American Institute of Mining, Metallurgical, and Petroleum Engineers, p. 370-399.

Guild, P. W., 1981, Preliminary metallogenic map of North America: numerical listing of deposits: U.S. Geological Survey Circular 858-A, $93 \mathrm{p}$.

Hall, James, 1843, Geology of New York, Part IV, Survey of the Fourth Geological District: Albany, N.Y., 683 p.

Hall, W. E., and Friedman, Irving, 1963, Composition of fluid inclusions, Cave-in-Rock fluorite district, Illinois, and upper Mississippi Valley zinc-lead district: Economic Geology, v. 58, p. 886-911.

Harris, L. D., 1969, Kingsport Formation and Mascot Dolomite (Lower Ordovician) of East Tennessee: Tennessee Division of Geology Report of Investigations 23, p. 1-39.

1971, A lower Paleozoic paleoaquifer, the Kingsport Formation and Mascot Dolomite of Tennessee and southwest Virginia: Economic Geology, v. 66, no. 5, p. 735-743.

Hartnagel, C. A., and Broughton, J. G., 1951, The mining and quarry industries of New York State, 1937 to 1948: New York State Museum Bulletin 343, $130 \mathrm{p}$.

Hatch, J. R., 1983, Geochemical processes that control minor and trace element composition of United States coals, in Shanks, W. C., III, ed., Cameron volume on unconventional mineral deposits: New York Society of Mining Engineers of the American Institute of Mining, Metallurgical, and Petroleum Engineers, Inc., p. 89-98.

Hayes, L. G., 1960, The Bowers-Campbell mine [Virginia]-Tri-State's boot-shaped zinc deposit: Mining Engineering, v. 12, no. 9, p. 9971000.

Heck, E. T., 1940a, Devonian coal in Tucker County, West Virginia: West Virginia Academy of Science Proceedings, Series 40, v. 13, no. 10-I, p. 81-82.

1940b, Barium in Appalachian salt brines: American Association of Petroleum Geologists Bulletin, v. 24, no. 3, p. 486492.

Heckel, P. H., 1973, Nature, origin, and significance of the Tully limestone: Geological Society of America Special Paper 138, $244 \mathrm{p}$.

Heinrich, E. W., 1976, The Mineralogy of Michigan: Michigan Geological Survey Bulletin 6, $225 \mathrm{p}$.

Herbert, Paul, Jr., and Young, R. S., 1956, Sulfide mineralization in the Shenandoah Valley of Virginia: Virginia Division of Geology Bulletin $70,58 \mathrm{p}$. 
Heyl, A. V., Jr., 1968a, The Upper Mississippi Valley base-metal district, in Ridge, J. D., ed., Ore deposits of the United States, 1933-1967, v. 1: New York, American Institute of Mining, Metallurgical, and Petroleum Engineers, p. 431-459.

1968b, Minor epigenetic, diagenetic, and syngenetic sulfide, fluorite, and barite occurrences in the central United States: Economic Geology, v. 63, no. 6, p. 585-594.

1982, Outlying mineral occurrences related to the Upper Mississippi Valley mineral district, Wisconsin, lowa, Illinois, and Minnesota: Economic Geology, v. 77, no. 8, p. 1803-1817.

1972, The 38th parallel lineament and its relationship to ore deposits: Economic Geology, v. 67, no. 7, p. 879-894.

1983, Geologic characteristics of three major Mississippi Valley districts, in Kisrarsayi, Geza, Grant, S. K., Pratt, W. P., and Koenig, J. W., ed., International Conference on Mississippi Valley type lead-zinc deposits: Rolla, Missouri, University of Missouri-Rolla, p. $27-60$

Heyl, A. V., Jr., Agnew, A. F., Lyons, E. J., and Behre, C. H., Jr., 1959 The geology of the Upper Mississippi Valley zinc-lead district: U.S. Geological Survey Professional Paper 309, 310 p.

Heyl, A. V., Jr., and Brock, M. R., 1962, Zinc occurrence in the Serpent Mound structure of southern Ohio: U.S. Geological Survey Professional Paper 450-D, p. D95-D97.

Heyl, A. V., Jr., Brock, M. R., Jolly, J. L., and Wells, C. E., 1965, Regional structure of southeast Missouri and Illinois-Kentucky mineral districts: U.S. Geological Survey Bulletin 1202-B, p. B1B20.

Heyl, A. V., Jr., Landis, G. P., and Zartman, R. E., 1974, Isotopic evidence for the origin of Mississippi Valley-type mineral deposits- $a$ review: Economic Geology, v. 69, no. 6, p. 992-1006.

Heyl, A. V., Jr., and Pearre, N. C., 1965, Copper, zinc, lead, iron, cobalt, and barite deposits in the Piedmont upland of Maryland: Maryland Geological Survey Bulletin 28, 72 p.

Heyl, A. V., Jr., and West, W. S., 1982, Outlying mineral occurrences related to the upper Mississippi Valley mineral district, Wisconsin, Iowa, and Minnesota: Economic Geology, v. 77, no. 8, p. 1803 1817.

Hill, W. T., 1969, Mine geology of the New Jersey Zinc Company's Flat Gap Mine at Treadway in the Copper Ridge district: Tennessee Division of Geology Report of Investigations 23, p. 76-90.

Hill, W. T., McCormick, J. E., and Wedow, Helmuth, Jr., 1971, Problems on the origin of ore deposits in the Lower Ordovician formations of East Tennessee: Economic Geology, v. 66, no. 5, p. 799-804.

Hoagland, A. D., 1962, Distribution of zinc in soils overlying the Flat Gap mine: Society of Mining Engineers Transactions, v. 223, no. 4, p. 399-402.

1967, Interpretations relating to the genesis of east Tennessee zinc deposits: Economic Geology Monograph 3, p. 52 58 .

Hoagland, A. D., Hill, W. T., and Fulweiler, R. E., 1965, Genesis of the Ordovician zinc deposits in east Tennessee: Economic Geology, v. 60 , no. 4 , p. $693-714$

Holden, W. F., and Carlson, E. H., 1979, Barite concretions from the Cleveland Shale in north-central Ohio: Ohio Journal of Science, $v$. 79 , no. 5 , p. $227-232$.

Hollenbaugh, D. W., and Carlson, E. H., 1983, The occurrence of wurtzite polytypes in eastern Ohio: Canadian Mineralogist, v. 21, p 697-703.

Howard, C. L. H., 1959, Celestite and fluorite from Clay Center, Ohio: Cleveland Museum of Natural History, Museum News, v. 1, p. 125130

Hughes, T. H., and Lynch, R. E., Jr., 1973, Barite in Alabama: Geological Survey of Alabama Circular 85, 43 p.

Hull, J. P. D., 1920, Report on the barytes deposits of Georgia: Geological Survey of Georgia Bulletin 36, $146 \mathrm{p}$

Hurst, V. J., and Crawford, T. J., 1970, Sulfide deposits in the Coosa Valley area, Georgia: Washington, D. C., U.S. Department of Commerce Economic Development Administration, $190 \mathrm{p}$.

Hyde, C., and Landy, R. A., 1966, Whewellite from septarian concretions near Milan, Ohio: The American Mineralogist, v. 51, p. 228-229.
Ingham, A. I., 1940, The zinc and lead deposits of Shawangunk Mountain, New York: Economic Geology, v. 35, p. 751-760.

Jacobs, E. C., 1943(?), Report of the State Geologist on the mineral industries and geology of Vermont, 1941-1942: Vermont Geological Survey, State Geologist 23rd Report, 83 p.

1944(?), Report of the State Geologist on the mineral industries and geology of Vermont, 1943-1944: Vermont Geological Survey, State Geologist 24th Report, p. 38-41.

Jensen, D. E., 1942, Minerals of the Lockport dolomite in the vicinity of Rochester, New York: Rocks and Minerals, v. 17, p. 199-203.

Jewell, W. B., 1947. Barite, fluorite, galena, sphalerite veins of middle Tennessee: Tennessee Department of Conservation, Division of Geology Bulletin 51, $114 \mathrm{p}$

Johnson, H. E., 1961, (letter) A hugh septarium from West Virginia: Rocks and Minerals, November-December, p. 581.

Jolly, J. L., and Heyl, A. V., Jr., 1964, Mineral paragenesis and zoning in the central Kentucky mineral district: Economic Geology, v. 59, no. 4 , p. $596-624$

Jones, W. B., and McVay, T. N., 1934, Barite deposits of the Sinks district, Bibb County, Alabama: Economic Geology, v. 29, p. 761766.

Kain, J. H., 1818, Remarks on the mineralogy and geology of the northwestern part of the State of Virginia and the eastern part of the State of Tennessee: American Journal of Science, v. 1, p. 60-67.

Kendall, D. L., 1960, Ore deposits and sedimentary features, Jefferson City mine, Tennessee: Economic Geology, v. 55, no. 5, p. 9851003.

Kesler, T. L., 1950, Geology and mineral deposits of the Cartersville district, Georgia: U.S. Geological Survey Professional Paper 224, $97 \mathrm{p}$.

King, P. B., Ferguson, H. W., Craig, L. C., and Rodgers, John, 1944 Geology and manganese deposits of northeastern Tennessee: Tennessee Department of Conservation, Division of Geology Bulletin 52, $283 \mathrm{p}$

King, P. B., and Ferguson, H. W., 1960, Geology of northeasternmost Tennessee: U.S. Geological Survey Professional Paper 311, $136 \mathrm{p}$.

King, P. B., and Beikman, H. M., (compilers) 1974, Geologic map of the United States: U.S. Geological Survey, scale 1:2,500,000.

Kinsland, G. L., 1977, Formation temperature of fluorite in the Lockport Dolomite in Upper New York State as indicated by fluid inclusion studies - with a discussion of heat sources: Economic Geology, v. 72 , no. 5 , p. $849-854$.

Kisvarsanyi, Geza, Grant, S. K., Pratt, W. P., and Koenig, J. W., ed., 1983, International conference on Mississippi Valley type lead-zinc deposits: Rolla, Missouri, University of Missouri-Rolla, $603 \mathrm{p}$.

Klemic. Harry, Warman, J. C., and Taylor, A. R., 1963, Geology and uranium occurrences of the northern half of the Lehighton, Pennsylvania, quadrangle and adjoining areas: U.S. Geological Survey Bulletin 1138, 97 p.

Kyle, J. R., 1976, Brecciation, alteration and mineralization in the Central Tennessee zinc district: Economic Geology, v. 71, no. 5, p. 892 903

Lane, A. C., 1900, Geological Report on Huron County, Michigan: Michigan Geological Survey, Report 7, pt. II, 329 p.

Lapham, D. M., and Geyer, A. R., 1959, Mineral collecting in Pennsylvania: Pennsylvania Geological Survey Bulletin G 33, 148 p.

Laurence, R. A., 1960, Geologic problems in the Sweetwater barite district, Tennessee: American Journal of Science, v. 258-A, p. 170 179.

Leavens, P. B., 1968, New data on Whewellite: The American Mineralogist, v. 53, p. 455-463.

Lechler, P. J., and others, 1979, Distribution and geochemical characterization of the Hannibal Member of the New Albany Shale in Indiana: Proceedings of the Third Eastern Gas Shales Symposium, Morgantown, West Virginia, U.S. Department of Energy METC/SP7916, p. 511-525

Lechler, P. J., Leininger, R. K., Shaffer, N. R., and Ripley, E. M., 1980 Enrichment of heavy metals in the New Albany Shale, southern Indiana (abs.): Geological Society of America Abstracts with Programs, v. 12 , no. 5 , p. 232-249. 
Leeds, A. R., 1875, On an asphaltic coal from the shale of the Huron River, Ohio, containing seams of sulphate of baryta: Lyceum of Natural History of New York, Annals, v. 11, p. 105.

Lesure, F. G., 1957, Geology of the Clifton Forge iron district, Virginia: Virginia Polytechnic Institute Engineering Experiment Station Series Bulletin 118, $130 \mathrm{p}$.

Lesure, F. G., and Klemic, Harry, 1977, Favorable area for zinc exploration, Saratoga County, New York: U.S. Geological Survey Open-File Report 77-319, 4 p.

Lesure, F. G., Williams, B. B., and Dunn, M. L., Jr., 1982, Mineral resources of the Mill Creek, Mountain Lake, and Peters Mountain Wilderness Study Areas, Craig and Giles Counties, Virginia, and Monroe County, West Virginia: U.S. Geological Survey Bulletin $1510,76 \mathrm{p}$.

Ludlum, J. C., 1955, Regional setting and mineralogic features of the Howell zinc prospect, Jefferson County, West Virginia: Economic Geology, v. 50, no. 8, p. 855-861.

Luttrell, G. W., 1966, Base- and precious-metal and related ore deposits of Virginia: Virginia Division of Mineral Resources, Mineral Resources Report 7, $167 \mathrm{p}$.

Maher, S. W., 1958, The zinc industry of Tennessee: Tennessee Department of Conservation, Division of Geology Information Circular no. 6, $28 \mathrm{p}$.

1970, Barite resources of Tennessee: Tennessee Department of Conservation, Division of Geology Report of Investigation 28, 40 $\mathrm{p}$.

Maher, S. W., and Finlayson, C. P., 1965, Investigations of miscellaneous mineral deposits in east Tennessee: Tennessee Academy of Science Joutnal, v. 40 , no. 2 , p. $43-47$.

Main, F. H., 1976, Zinc in Tennessee: New Jersey Zinc's contribution, in Appalachian Regional Commission, Proceedings of the Appalachian Mineral Resources Evaluation Conference, p. 113-121.

Martens, J. H. C., 1925, Barite and associated minerals in concretions in the Genesee Shale [central New York]: American Mineralogist, v. 10 , p. $102-104$.

1963, Sulphate minerals in West Virginia: West Virginia Geological and Economic Survey Bulletin 25, 13 p

1964, Minerals of West Virginia: West Virginia Geological and Economic Survey Educational Series 8, $41 \mathrm{p}$.

Mather, W. W., 1843, Geology of New-York, Part 1, Comprising the geology of the First Geological District: Albany, N.Y., 653 p.

McAlister, R. F., 1946, Famous Clay Center Ohio locality: The Mineralogist, v. 14 , p. $623-624$.

McCormick, J. E., Evans, L., L., Palmer, R. A., and Rasnick, F. D., 1971 Environment of the zinc deposits of the Mascot-Jefferson City district Tennessee: Economic Geology, v. 66, no. 5, p. 757-762.

McLimans, R. K., 1977, Geological, fluid inclusion, and stable isotope studies of the upper Mississippi Valley zinc-lead district, southwest Wisconsin: University Park, Pa., The Pennsylvania State University, Ph. D. dissertation, $187 \mathrm{p}$

McLimans, R. K., Barnes, H. L., and Ohmoto, H., 1980, Sphalerite stratigraphy of the upper Mississippi Valley zinc-lead district, southwestern Wisconsin: Economic Geology, v. 75, no. 3, p. 351361.

Merrill, F. J. H., 1895, Mineral resources of New York State: New York State Museum Bulletin 15, p. 363-595.

Miller, W. J., 1910, Geology of the Port Leyden quadrangle, Lewis County, New York: New York State Museum Bulletin 135, 61 p.

Montague, J. O., 1948, Clay Center, Ohio: The Mineralogist, v. 16, June-July issue, p. 300-302.

Morrill, Philip, and Chaffee, R. G., 1964, Vermont mines and mineral localities: Hanover, New Hampshire, Dartmouth College Museum, $57 \mathrm{p}$.

Morrison, R. B., 1935, The occurrence and origin of celestite and fluorite at Clay Center, Ohio: American Mineralogist, v. 20, p. 780-790.

Multer, H. G., 1963, Geology of the Silurian producing zones in the Moreland oil pool, Wayne County, northeastern Ohio: Ohio Geological Survey Report of Investigations 46, 48 p.
Napper, C. W., 1917, Concretionary forms in the Greenfield limestone: Ohio Journal of Science, v. 18, p. 7-13.

Neatherly, T. L., Clarke, O. M., Jr., Smith, W. E., Szabo, M. W., and White, D. H., Jr., 1972, Mineral resources of Calhoun County, Alabama: Alabama Geological Survey Circular 81, p. 21-24.

Neumann, G. L., 1952a, Lead-zinc deposits of southwestern St. Lawrence County, N.Y.: U.S. Bureau of Mines Report of Investigations $4907,25 \mathrm{p}$.

1952b, Guymard lead-zinc deposit, Orange County, N.Y.: U.S. Bureau of Mines Report of Investigations 4909, $10 \mathrm{p}$.

Newland, D. H., 1919, The mineral resources of the State of New York: New York State Museum Bulletins 223 and 224, 315 p.

Noble, E. A., 1963, Formation of ore deposits by water of compaction: Economic Geology, v. 58, no. 7, p. 1145-1156.

Oder, C. R. L., and Ricketts, J. E., 1961, Geology of the MascotJefferson City zinc district, Tennessee: Tennessee Department of Conservation, Division of Geology Report of Investigations 12, $29 \mathrm{p}$.

Ohle, E. L., 1959, Some considerations in determining the origin of ore deposits of the Mississippi Valley type: Economic Geology, v. 54, no. 5, p. 769-789.

1980 , Some considerations in determining the origin of ore deposits of the Mississippi Valley type-Part II: Economic Geology, v. 75 , no. 2 , p. $161-172$.

Ostrander, C. W., 1942, Barite prospect near Johnsville, Frederick County, Maryland: Natural History Society of Maryland Bulletin, v. 12 , no. 3 , p. 44 .

Parr, D. F., and Chang, L. L. Y., 1977, Descriptive mineralogy of Pugh Quarry, northwestern Ohio-marcasite and pyrite: Ohio Journal of Science, v. 77, p. 213-222.

1978, Descriptive mineralogy of Pugh Quarry, northwestern Ohio-sphalerite: Ohio Journal of Science, v. 78, p. 272-279.

1979, Descriptive mineralogy of Pugh Quarry, northwestern Ohio-calcite, dolomite, and fluorite: Ohio Journal of Science, v. 79, p. 24-31

1980, Descriptive mineralogy of Pugh Quarry, northwestern Ohio-barite and celestite: Ohio Journal of Science, v. 80, p. 20-29.

Payne, J. N., 1938, Discovery of sphalerite and galena near Millbrook, Kendall County: Illinois Academy of Science Transactions, v. 31, no, 2, p. $182-183$.

Pepper, J. 'F., Clark, S. H. B., and deWitt, Wallace Jr., 1985, Spherulitic nodules of barite in Late Devonian shales of western New York: U.S. Geological Survey Bulletin 1653, 11 p.

Peterson, R. B., 1950, The mineral industries of New York State: Albany, New York State Department of Commerce, $108 \mathrm{p}$.

Pinckney, D. M., 1976, Mineral resources of the Illinois-Kentucky mining district: U.S. Geological Survey Professional Paper 970, 15 p.

Plummer, L. N., 1971, Barite deposition in Central Kentucky: Economic Geology, v. 66, no. 2, p. 252-258.

Pratt, W. P., 1984, Mississippi Valley-type lead-zinc deposits, Conference report: Geology, v. 12 , no, 3 , p. 184

Price, P. H., Hare, C. E., McCue, J. B., and Hoskins, H. A., 1937, Salt brines of West Virginia: West Virginia Geological Survey Report, v. $8,203 \mathrm{p}$.

Ramsey, J. G. M., 1926, The annals of Tennessee: Kingsport, Kingsport Press, 2nd ed., p. 376

Reade, E. H., Power, W. R., Keeners, M. J., White, D. H., 1980, Barite deposits in the Cartersville District, Bartow County, Georgia, in Robert Frey, ed., Excursions in southeastern geology, v. II: American Geological Institute Guidebook for Geological Society of America 1980 Annual Meeting, p. 379-384.

Reger, D. B., and Tucker, R. C., 1924, Mineral and Grant Counties: West Virginia Geological Survey, 866 p.

Reidel, S. P., 1975, Bedrock geology of the Serpent Mound cryptoexplosion structure, Adams, Highland, and Pike Counties, Ohio: Ohio Geological Survey Report of Investigations 95, 1 sheet with text.

Reidel, S. P., and Koucky, F. L., 1981, The Serpent Mound cryptoexplosion structure, southwestern Ohio, in GSA Cincinnati '81 Field Trip Guidebooks, v. 2: Falls Church, Virginia, American Geological Institute, p. 391-403. 
Robinson, L. C., 1931, Vein deposits of central Kentucky: Kentucky Geological Survey, Series 6, v. 41, 127 p.

Rodgers, John, 1948, Geology and mineral deposits of Bumpass Cove, Unicoi and Washington Counties, Tennessee: Tennessee Department of Conservation, Division of Geology Bulletin 54, $82 \mathrm{p}$.

Roedder, Edwin, 1967, Environment of deposition of stratiform (Mississippi Valley-type) ore deposits, from studies of fluid inclusions: Economic Geology Monograph 3, p. 349-362.

1976, Fluid-inclusion evidence on the genesis of ores in sedimentary and volcanic rocks, in Wolf, K. N., ed. Handbook of stratabound and stratiform ore deposits: Amsterdam, Elsevier Scientific Publishing Company, Amsterdam, p. 67-110.

Rogers, J. K., 1936, Geology of Highland County: Ohio Geological Survey, Fourth Series, Bulletin 38, 148 p.

Rominger, C. L., 1876, Lower peninsula 1873-1876, Geology of lower peninsula, Michigan: Geological Survey of Michigan Reports, v. III, pt. $1,150 \mathrm{p}$.

Rose, A. W., 1970, Metal mines and occurrences in Pennsylvania: Pennsylvania Geological Survey Bulletin M 50, part 3, 14 p.

Sangster, D. F., 1983, Mississippi Valley-type lead-zinc deposits: a geological melange, in Kisvarsanyi, Geza, Grant, S. K., Pratt, W. P., and Koenig, J. W., ed., International conference on Mississippi Valley type lead-zinc deposits: Rolla, Missouri, University of MissouriRolla, p. 7-19.

Sayrs, R. L., and Clayton, A. B., 1949, Investigations of Idol and Dalton zinc deposits, Grainger County, Tennessee: U.S. Bureau of Mines Report of Investigations 4497, $7 \mathrm{p}$.

Schrode, R. S., 1952, Barite in the LaSalle Limestone of Illinois: Illinois Academy of Science Transactions, (1951), v. 44, p. 126-128; Illinois Geological Survey Circular 179, p. 126-128.

Seaman, D. M., and Hamilton, Howard, 1950, Occurrence of polymorphous wurtzite in western Pennsylvania and eastern Ohio: American Mineralogist, v. 35, p. 43-50.

Secrist, M. H., 1924, Zinc deposits of east Tennessee: Tennessee Department of Conservation, Division of Geology Bulletin 31, $165 \mathrm{p}$.

Shaffer, N. R., 1981, Possibility of Mississippi Valley-type mineral deposits in Indiana: Indiana Geological Survey Special Report 21, $49 \mathrm{p}$.

Shaffer, N. R., Leininger, R. K., Ripley, E. M., and Gilstrap, M. S., 1981, Heavy metals in organic-rich New Albany Shale of Indiana (abs): Geological Society of America Abstracts with Programs, v. 13, p. 551.

Sims, P. K., and Hotz, P. E., 1951, Zinc-lead deposit at Shawangunk mine, Sullivan County, New York: U.S. Geological Survey Bulletin 978-D, p. 101-121.

Smith, R. C., II, 1974, Fetid barite from Berks County, Pennsylvania: Pennsylvania Geology, v. 5, no. 6, p. 4-7.

1977, Zinc and lead occurrences in Pennsylvania: Pennsylvania Geological Survey Mineral Resources Report 72, $318 \mathrm{p}$.

Smith, R. C., II, Herrick, D. C., Rose, A. W., and McNeal, J. M., 1971, Zinc-lead occurrences near Mapleton, Huntingdon County, Pennsylvania: The Pennsylvania State University Earth and Mineral Sciences Experimental Station Circular 83, 36 p.

Snyder, F. G., 1968, Geology and mineral deposits, midcontinent United States, in Ridge, J. D., ed., Ore deposits of the United States, 19331967, v. 1: New York, American Institute of Mining, Metallurgical, and Petroleum Engineers, p. 255-286.

Socolow, A. A., 1959, Geology of a barite occurrence, Fulton County, Pennsylvania: Pennsylvania Topographic and Geological Survey Information Circular 17, $5 \mathrm{p}$.

Stone, R. W., 1939, Non-metallic minerals, in The minerals of Pennsylvania: Pennsylvania Geological Survey Bulletin M 18-C, $46 \mathrm{p}$.

Stose, A. I. J., and Stose, G. W., 1957, Geology and mineral resources of the Gossan lead district and adjacent areas in Virginia: Virginia Division of Mineral Resources Bulletin 72, 233 p.

Stout, W. E., 1941, Dolomites and limestones of western Ohio: Ohio Geological Survey, Fourth Series, Bulletin 42, 468 p.
Strong, Moses, 1882, Geology of the Mississippi region north of the Wisconsin River: [Wisconsin Geological Survey], Geology of Wisconsin Survey of 1873-1879, v. 4, p. 3-98.

Sverjensky, D. A., 1981, The origin of a Mississippi Valley-type deposit in the Viburnum Trend, southeast Missouri: Economic Geology, v. 76, no. 7, p. $1848-1872$.

Sverjensky, D. A., Rye, R. O., and Doe, B. R., 1979, The lead and sulfur isotope compositions of a galena from a Mississippi Valley-type deposit in the New Lead Belt, Southeast Missouri: Economic Geology, v. 74, no. 1, p. 149-153.

Sweet, P. C., and Rowe, W. D., 1984: Selected Virginia mineral-resource information: Virginia Division of Mineral Resources Publication 51, $28 \mathrm{p}$.

Swingle, G. D., 1959, Geology, mineral resources, and ground water of the Cleveland area, Tennessee: Tennessee Division of Geology Bulletin 61, $125 \mathrm{p}$.

Taylor, A. R., 1962, Geology of the Amandaville Quadrangle, Kentucky: U.S. Geological Survey Geologic Quadrangle Map GQ-186, scale $1: 24,000$.

Trace, R. D., 1976, Illinois-Kentucky fluorspar district, in Shaw, D. R., ed., Geology and resources of fluorine in the United States: U.S. Geological Survey Professional Paper 933, p. 63-72.

Ver Steeg, Karl, 1940, Sphalerite and galena in sedimentary rocks in Ohio: Science, v. 92, p. 259. p. 223.

1942, Galena in concretions of Pottsville age: Science, v. 95,

Watson, T. L., 1905, Lead and zinc deposits of Virginia: Geological Survey of Virginia, Bulletin 1, $156 \mathrm{p}$.

1907, Mineral resources of Virginia, Lynchburg, Virginia: J. P. Bell Company, p. 520-548.

Watson, T. L., and Grasty, J. S., 1915, Barite of the Appalachian States: American Institute of Mining Engineers Bulletin 98, p. 345-390.

Way, J. H., and Smith, R. C. II, 1983, Barite in the Devonian Marcellus Formation, Montour County: Pennsylvania Geology, v. 14, no. 1, p. 4-9.

Weinberg, E. L., 1971, Sulfide mineralization, southwestern Virginia: Blacksburg, Virginia, Virginia Polytechnic Institute and State University Department of Geological Sciences, Guidebook no. 5, Blacksburg, Virginia, p. 6-23.

White, D. E., 1958, Liquid of inclusions in sulfides from Tri-State (Missouri-Kansas-Oklahoma) is probably connate in origin (abs.): Geological Society of America Bulletin, v. 69, p. 1660-1661.

1968 , Environments of generation of some base-metal ore deposits: Economic Geology, v. 63, no. 4, p. 301-335.

1974, Diverse originis of hydrothermal ore fluids: Economic Geology, v. 69, p. 954-973.

White, Lane, 1979, Middle Tennessee zinc; Jersey Miniere plans growth at Elwood-Gordonsville Mine: Engineering and Mining Journal, v. 180 , no. 8 , p. $66-76$.

Wedow, Helmuth, Jr., 1983, A search for stratiform massive-sulfide exploration targets in Appalachian Devonian rocks - a case study using computer-assisted attribute-coincidence mapping: U.S. Geological Survey Open-File Report 83-352, 82 p.

Williams, J. S., Duncan, Helen, Hardin, G. C., Jr., Trace, R. D., Thurston, W. R., and Klepser, H. J., 1954 (1955), Fluorspar deposits in western Kentucky: U.S. Geological Survey Bulletin 1012, 127 p.

Willman, H. B., and Reynolds, R. R., and Herbert, Paul, Jr., 1946, Geological aspects of prospecting and areas for prospecting in the zinc-lead district of northwestern Illinois: Illinois Geological Survey Report of Investigation 116, 48 p.

Windolph, J. F., Jr., 1985a, Geologic map of the Amonate Quadrangle, Virginia-West Virginia: U.S. Geological Survey Geologic Quadrangle Map GQ-1597.

1985b, Maps showing coal resources of the Amonate Quadrangle, Buchanan and Tazewell Counties, Virginia and McDowell County, West Virginia: U.S. Geological Survey Miscellaneous Field Studies Map MF-1730.

Winslow, K. R., and Hill, W. T., 1973, The Elmwood project: Mining Congress Journal, v. 59, no. 3, p. 19-27. 
Woodward, H. P., 1932, Geology and mineral resources of the Roanoke area, Virginia: Virginia Geological Survey Bulletin 34, $172 \mathrm{p}$.

1938, Outline of the geology and mineral resources of Russell County, Virginia: Virginia Geological Survey Bulletin 49, $91 \mathrm{p}$.

Worl, R. G., Van Alstine, R. E., and Heyl, A. V., 1974, Fluorite in the United States, exclusive of Hawaii: U.S. Geological Survey Mineral Investigations Resource Map MR-60, 13 p., map.

Young, R. S., 1956, Sulfides in Virginia: Virginia Division of Geology, Virginia Minerals, v. 2, no. 1, p. 1-7.

1967, Reconsideration of the Shenandoah Valley sulfide district, Virginia (abs.): Virginia Journal of Science, v. 18, no. 4, p. 190.

Zodac, Peter, 1947, Trip to Detroit: Rocks and Minerals, v. 22, p. $1011-$ 1017.

Zubovic, P., 1960, Minor element content of coal from Illinois beds 5 and 6 and their correlatives in Indiana and western Kentucky: U.S. Geological Survey Open-File Report, 79 p. 


